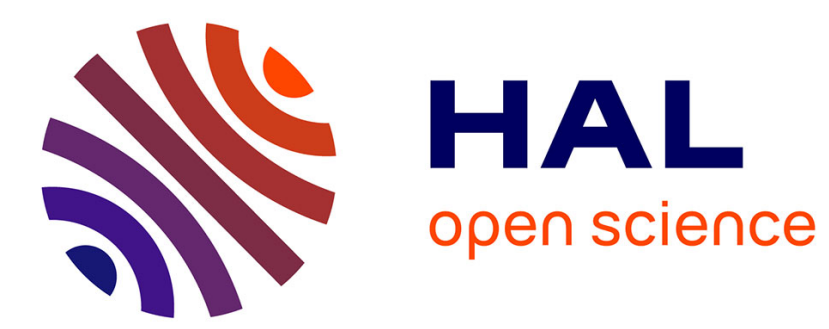

\title{
How to face the complexity of plasmas?
}

Dominique Escande

\section{To cite this version:}

Dominique Escande. How to face the complexity of plasmas?. 2012. hal-00702276v3

\section{HAL Id: hal-00702276 \\ https://hal.science/hal-00702276v3}

Preprint submitted on 12 Jul 2012

HAL is a multi-disciplinary open access archive for the deposit and dissemination of scientific research documents, whether they are published or not. The documents may come from teaching and research institutions in France or abroad, or from public or private research centers.
L'archive ouverte pluridisciplinaire HAL, est destinée au dépôt et à la diffusion de documents scientifiques de niveau recherche, publiés ou non, émanant des établissements d'enseignement et de recherche français ou étrangers, des laboratoires publics ou privés. 


\title{
How to face the complexity of plasmas?
}

\author{
D. F. Escande \\ UMR 7345 CNRS-Aix-Marseille-Université, Facultée de St Jérôme, case 321, \\ Av. Normandie Niemen, FR-13397 Marseille CEDEX 20*
}

\begin{abstract}
This paper has two main parts. The first part is subjective and aims at favoring a brainstorming in the plasma community. It discusses the present theoretical description of plasmas, with a focus on hot weakly collisional plasmas. It comprises two sub-parts. The first one deals with the present status of this description. In particular, most models used in plasma physics are shown to have feet of clay, there is no strict hierarchy between them, and a principle of simplicity dominates the modeling activity. At any moment the description of plasma complexity is provisional and results from a collective and somewhat unconscious process. The second sub-part considers possible methodological improvements, some of them specific to plasma physics, some others of possible interest for other fields of science. The proposals for improving the present situation go along the following lines: improving the way papers are structured and the way scientific quality is assessed in the referral process, developing new data bases, stimulating the scientific discussion of published results, diversifying the way results are made available, assessing more quality than quantity, making available an incompressible time for creative thinking and non purpose-oriented research. Some possible improvements for teaching are also indicated. The suggested improvement of the structure of papers would be for each paper to have a "claim section" summarizing the main results and their most relevant connection to previous literature. One of the ideas put forward is that modern nonlinear dynamics and chaos might help revisiting and unifying the overall presentation of plasma physics.

The second part of this chapter is devoted to one instance where this idea has been developed for three decades: the description of Langmuir wave-electron interaction in one-dimensional plasmas by a finite dimensional Hamiltonian. This part is more specialized, and is written like a classical scientific paper. This Hamiltonian approach enables recovering Vlasovian linear theory with a mechanical understanding. The quasilinear description of the weak warm beam is discussed, and it is shown that self-consistency vanishes when the plateau forms in the tail distribution function. This leads to consider the various diffusive regimes of the dynamics of particles in a frozen spectrum of waves with random phases. A recent numerical simulation showed that diffusion is quasilinear when the plateau sets in, and that the variation of the phase of a given wave with time is almost non fluctuating for random realizations of the initial wave phases. This led to new analytical calculations of the average behavior of the self-consistent dynamics when the initial wave phases are random. Using Picard iteration technique, they confirm numerical results, and exhibit a spontaneous emission of spatial inhomogeneities.
\end{abstract}

Non quia difficilia sunt, non audemus, sed quia non audemus, difficilia sunt.

It is not because things are difficult that we do not dare, it is because we do not dare that things are difficult. [Seneca, Epistulae morales 104, 26]

\section{INTRODUCTION}

One of the points of this chapter is that plasma physics, and probably also other fields of science would benefit from a modification in the structure of scientific papers: each new paper would come with an "executive summary", longer than an abstract, providing its main results and its most relevant references. This idea is developed in section II B 1 for research papers. Since this chapter must take on the usual structure of scientific papers and must start with a classical introduction, an extended summary is provided in Appendix 1.

This introduction is split into two parts: the introduction to the contents of the chapter a nd a short introduction to plasma physics for non experts. 


\section{A. What this chapter is about}

For Aristotle, the study of human knowledge called theoria ("contemplation") was the highest human knowledge and happiness. This chapter is inspired by this kind of philosophy. Indeed, its first part aims at discussing the present way plasmas are theoretically described, with a focus on hot weakly collisional plasmas. It states views that are biased by the author's personal research background ${ }^{1}$, but which are in the spirit of favoring a collective brainstorming about how to deal with plasma complexity. Indeed, in agreement with what is told in section II A 3, an authoritative review about plasma complexity should be written by a series of committees of experts, as was already done twice for ITER physics basis [81, 127]. The first part of this chapter (section II) comprises two main sub-parts. The first one (section II A) deals with the present status of this description. The second one (section II B) considers possible methodological improvements, some of them specific to plasma physics, some others of possible interest for other fields of science. To the best of the author's knowledge such a work has not yet been done, while it might be useful for the development of plasma physics. Indeed, till now this physics has developed in a somewhat continuous way. This shows up in several ways. As to textbooks about plasma physics with a broad scope, modern ones provide synthetic views, but they do not fundamentally challenge the contents of previous ones. As to research programs, they are strongly purpose oriented, which does not help theoreticians to stop and to look backward. This is very much the case for the research on thermonuclear fusion by magnetic confinement. Indeed for more than five decades the fusion reactor has been thought as coming soon ${ }^{2}$.

Therefore one of the purposes of this paper is to stop and to look backward for proceeding better ahead. How do we work? How could our community improve its methodology in order to improve its efficiency, and to get more satisfaction and even more joy in its practice? Although we will discuss general issues and possible ways to improve the present understanding of complex systems (in particular of plasmas), for the sake of definiteness working examples will be taken from the author's direct research experience in hot plasmas. One of the ideas put forward in section II B is that modern nonlinear dynamics and chaos might help revisiting and unifying the overall presentation of plasma physics.

The second part of this chapter (section III) is more specialized, and is devoted to one instance where this idea has been developed for three decades: wave-particle interaction in plasmas, and more specifically Langmuir wave-electron interaction in one-dimensional plasmas. In reality this second part corresponds to the author's invited talk at Chaos, Complexity and Transport 2011. Since this was an interdisciplinary conference, a short introduction to plasma physics is provided right after first giving a definition of "complexity" relevant to plasma physics.

"Complexity" is a word with a lot of meanings. The meaning used in this chapter is ubiquitous in modern science. However, this meaning comes with an increasing number of attributes when going from inanimate matter to living matter, to humans, and to societies. These attributes may be ordered in a sequence of levels of description. For inanimate matter the number of levels is smaller than for living systems. The coarsest level has two aspects: on the one hand the whole is more than its parts and on the second hand it displays a spontaneous self-organization. The former aspect may be very strong: the whole may be a lot beyond the sum of its parts, as occurs for open systems, be it a plasma column in a laboratory, or the human body whose matter is almost completely renewed about every two months through metabolism and repair. The latter aspect is important to tell complex systems from artifacts like computers or engines. It may come with two opposite, but possibly interrelated, features: order and chaos.

Emergence is the central feature of the level of description following the largest one. It results from self-organization, and is the appearance in the system of interest of a feature (form or pattern) arising out of a multiplicity of relatively simple interactions of smaller parts. This feature cannot be anticipated from the knowledge of the parts of the system alone, even if these parts are also complex systems made up of finer scales. A typical example of emergence structure is a fluid vortex, as occurring for instance due to the motion of water particles in a pipe flow. In turn, individual vortices may interact to produce another emergent feature: turbulence. This emergence makes the water less fluid than in the laminar state: pressure drop increases, but molecules are unaffected; again the whole is a lot beyond the sum of its parts.

In living systems, next levels of description include features like cooperation and competition. There are other definitions of complexity which introduce the same attributes in a different order, or other attributes which are implicitly present in the above definition: complex systems contain many interdependent constituents interacting

1 which is made explicit by a series of examples in the following.

2 The TFTR tokamak was shut down in 1997. For years before, people no longer insisted into calling it Tokamak Fusion Test Reactor. When the ITER project officially started in 1992, "ITER" meant "International Tokamak Experimental Reactor". Now "ITER" is the Latin noun meaning "the way" [127]. This is so true that the KTX machine, a large reversed field pinch, is being funded in Hefei in the frame of the Chinese ITER domestic program. 
nonlinearly, and their self-organization spans several spatial and temporal scales.

If a fluid or a plasma is described as an $N$-body system, its Hamiltonian is made up of the sum of all the free particle Hamiltonians, plus an interaction. Therefore the interaction is a part of the system. The same occurs when dealing with wave-particle interaction in plasmas. As will be shown in section III, the corresponding physics can be described by a Hamiltonian made up of a free particle part and a free wave (harmonic oscillator) part, plus a wave-particle interaction part. This description puts waves and particles on an equal footing, as occurs in modern field theory.

\section{B. Plasma physics}

A plasma is a quasineutral system of charged particles. The plasma state is often presented as the fourth state of matter, because it can be reached by further heating matter after it experiences successively the solid, liquid and gaseous states. With respect to these states, it has the distinctive feature of the long-range interaction of its particles because of the electromagnetic field they produce. This endows it with a ubiquitous collective behavior which shows up as waves, solitons, turbulent eddies, vortex structures, streamers, blobs, etc...

"Plasma" is a Greek word which means moldable, or without any definite shape. This lack of definite shape applies to plasma physics as well, because the plasma state is in reality contiguous to all three solid, liquid and gaseous states, which weakens its classification as the fourth state of matter in a one-dimensional classification. This is linked to the fact that this state may be realized with densities varying over more than 30 decades and temperatures varying over more than 7 decades. As a result there is a huge variety of plasma states, which sets complexity, and even complexities, at the very beginning of plasma theory. This chapter does not attempt to deal with this variety, but focuses on hot weakly collisional plasmas with relevance to astrophysics and especially thermonuclear fusion. Such plasmas are characterized by the fact that there are many particles in the Debye sphere, i.e. the sphere with a radius equal to the Debye length $\lambda_{\mathrm{D}}=\left[\left(\epsilon_{0} k_{\mathrm{B}} T\right) /\left(n e^{2}\right)\right]^{1 / 2}$ where $\epsilon_{0}$ is the vacuum permittivity, $k_{\mathrm{B}}$ is the Boltzmann constant, $T$ is the temperature, $n$ is the density, and $e$ is the electron charge. Therefore $n \lambda_{D}^{3} \gg 1$, which means $T^{3} / n \gg 1$ with appropriate normalizations.

The plasma state is the state of matter the most spread in the universe (except for dark matter!), and has many important applications. However, despite the obvious importance of the field and the extensive knowledge on plasmas, plasma physics has got a limited scientific recognition. No Nobel prize has been awarded to its physicists ${ }^{3}$. It was not quoted in the one hour opening talk "A century of physics" of the APS Centennial Meeting in 1999. This lack of scientific recognition may be partly related to the slower progress toward the thermonuclear reactor than expected. However this chapter provides another clue by showing the difficulties in describing plasmas. This difficulty makes the theory of plasmas more like an impressionist painting, than like a well structured theoretical knowledge. This chapter also shows the stimulating collective research process in plasma physics to be a hindrance for an individual to bring alone a decisive progress in plasma description. Plasma complexity is at the root of this situation. It is temporal as well as spatial. Indeed it stems from the many degrees of freedom nature of plasma dynamics, which exhibits a huge variety of dynamical modes. As Kadomtsev said "Here, similar to many paintings by the prominent artist Hieronymus Bosch, there exist many levels of perception and understanding. At a cursory glance of the picture you promptly grasp the idea. But under a more scrutinized study of its second and third levels you discover new horizons of a deeper life and it turns out that your first impressions become rather shallow" [82]. The "Garden of Earthly Delights" at the Madrid Prado museum is the author's favorite experience from this point of view ${ }^{4}$

\section{FACING PLASMA COMPLEXITY}

This section aims at favoring a brainstorming in the plasma community. It is made up of two parts. The first one deals with the present status of the theoretical description of plasmas, with a focus on hot weakly collisional plasmas. The second part considers possible methodological improvements, some of them specific to plasma physics, some others of possible interest for other fields of science. Since an authoritative review about plasma complexity should be the result of a collective effort, this section is inevitably subjective. Therefore some of its statements might possibly be unwillingly polemical.

\footnotetext{
3 Alfven's Nobel citation reads: (...) for fundamental work and discoveries in magnetohydrodynamics with fruitful applications in different parts of plasma physics. Therefore his award was not meant as presented to a plasma physicist. In reality, Alfven was in an awkward position with respect to well identified fields of physics [126].

${ }^{4}$ Increasing blow ups are advised when using Internet to watch a photograph of the painting.
} 


\section{A. Present status of the description of plasma complexity}

This section recalls the path used for student training, before dealing with the way theoreticians face complexity. It is recalled that models used in plasma physics, even the Vlasov equation, have feet of clay. Each plasma physicist is shown to elaborate his own global view about plasma physics from many models which do not have any strict hierarchy. The validation of assumptions turns out to be more difficult for a complex system than for a simple one. In agreement with Popper's falsifiability paradigm [105], at any moment the description of plasma complexity is provisional. It results from a collective and somewhat unconscious process. This makes changing views more difficult. Numerical simulations are discussed as a complex tool to face complexity.

What happens in this process is often similar to the Indian tale "The blind men and the elephant". Six blind men want to know what an elephant is, and go in the courtyard of the rajah's palace to touch one. Each of them touches a different part of the animal, and feels it is similar to an object he already knows: a wall, a spear, a snake, a tree, a fan, and a rope. They start arguing with one another so loudly that the rajah comes to his window, asks about the issue, and finally tells them they should put together all the pieces of information they got in order to know what an elephant is! Knowing what is a plasma comes with similar difficulties: the experimental knowledge is often scarce, but the theoretical views may be many. One must try and tie together the partial views of the experts. Though textbooks and review papers are of some help, the global knowledge about a given class of plasmas, e.g. magnetic fusion plasmas, is largely implicit, and each expert of the field has his own one ${ }^{5}$.

\section{Path for students}

Going from the simplest to the more complex in plasma physics, is a path any student must follow. At the roots of plasma physics there are basic textbook problems dealt with by elementary models. Two oppositely extreme ones are proposed to beginners: single particle and fluid models. Single particle dynamics comes under two main aspects: motion in a magnetic field with its corresponding drifts, and what is traditionally called "collisions" in plasma physics. Fluid models are introduced to describe plasmas as a whole, with two important aspects: ideal and resistive magnetohydrodynamics (MHD) on the one hand, and the description of waves and instabilities on the other hand. The knowledge of waves is of paramount importance because they are the simplest instance of the ubiquitous collective motions of plasmas, and because they are leading actors in plasma turbulence. Later on are introduced kinetic descriptions (Vlasov, gyrokinetic, etc... equations) which aim at describing plasmas as a whole while keeping single particle dynamics as much as possible into account. Textbooks describe basic phenomena with the simplest possible assumptions, like homogenous plasma either isotropic or in a uniform magnetic field. Then the intricacy of actual plasmas is introduced by taking into account various gradients of magnetic field, density, temperature, etc..., and new physics emerges corroborated by experimental results, which gives confidence into the models. However there are bad news.

\section{Models have feet of clay}

Indeed unfortunately most models have feet of clay! Indeed very few models used by plasma physicists are derived from first principles with assumptions that are justified for the class of experimental or theoretical problem of interest (an exception is presented in section III). Many models are derived under given assumptions, but, because of their handiness, they are used out of their domain of proved validity, or without knowing their limit of validity. Vlasov equation is considered as the reference equation for collisionless plasmas, but the derivation of this equation by a mean-field approach shows the validity of its solutions is proved up to the time of exponential divergence of nearby orbits (see for instance [115] for an introduction). For instance, this time a priori bounds the actual duration of the Bernstein-Green-Kruskal (BGK) solutions [14] of Vlasov equation. It is too stringent a time bound to describe turbulence, which is an incentive to keep plasma granularity for this purpose, as is done in section III. The latter approach reveals that an unstable plasma may reach a state away from the Vlasovian saturation state [66, 69], and that there are no BGK modes [45]: in reality a plasma state starting in a Vlasovian BGK state is metastable. Furthermore it was shown theoretically that a superthermal tail can be generated by beam-plasma interaction, which is missed by

5 An important exception is the ITER physics basis [81, 127], already mentioned in section I A, that was written by a series of committees of experts. This trend toward a collective view about the tokamak has been present in the authorship of Wesson's book [121] since its first edition in 1987. It is also worth noting that most modern plasma textbooks have at least two authors. 
a Vlasovian description [122]. The discrepancy of the Vlasovian solutions can be understood as a result of the non commutation of the infinite time and infinite number of particles limits. Indeed, consider the particles initially inside a given Debye sphere, and assume they have a chaotic motion. Later chaos stretches this sphere into a thin tube where these particles are far apart, which reveals that the plasma is no longer a fluid in phase space. Vlasov equation is also used for open systems, but its validity has not been proved formally (to the author's knowledge) in this case.

The use of basic fluid models is ubiquitous, but they cannot generally be justified from first principles for collisionless plasmas. For instance, the applicability of MHD equations to fusion plasmas may be justified if perpendicular motion dominates (see sections 2.5 of [17], 6 of [77], 3.5 of [78]), at least for the mass and momentum equations (see section 2.4 of $[71])$.

As Bertrand Russell stated, "Although this may seem a paradox, all exact science is dominated by the idea of approximation" [112]. This is all the more true in plasma physics, since the nature of approximations is not clear in many theoretical descriptions. Fortunately, very often the predictions they provide are found to have experimental relevance, or to be validated by more sophisticated models. This suggests that models have a larger validity than proved as yet, or that their equations describe the genuine evolution of quantities that are close to those explicitly present herein.

\section{A global view from many models without any strict hierarchy}

The typical complexity of plasmas rules out their description by the most general non quantum model which would account for all its particles, with possibly their finite lifetime inside the plasma, and its actual geometry. Therefore a whole fauna of "approximate" models must be used. Theoretical description and intuition develop by successive additions of complexity on the basis of the solution of a few simple problems, and of the confrontation to experiments and numerical simulations. The permanent irruption of new measurements and scientific interactions may bring a progressive complexification of a given model, or it may challenge it so much that a new one must be proposed. This evolution is often due to a community, and not to an individual.

Unfortunately, starting from the academic knowledge, there is no obvious path toward the more complex for the researcher, and in particular an axiomatic approach is generally formidable (an exception to this is provided in section III). Indeed complexity may be added to a simple model in many different ways. It may be by increasing dimensionality, or the complexity of geometry (from slab to cylindrical, and to toroidal), or the number of involved physical quantities, or the number of described species, or the number and type of transport coefficients, etc... One may go from fluid to kinetic descriptions, or from a linear to a nonlinear one, one may take inhomogeneities and fluctuations into account, etc... As plasma complexity has many dimensions, there is no strict hierarchy among these models.

A natural way to tackle a modeling issue is for the researcher to start by applying Occam's razor principle "Entities must not be multiplied beyond necessity", i.e. to choose the simplest available model in agreement with his present knowledge of the system of interest ${ }^{6}$. In the same spirit, he may state a priori what is the dominant physics, and build a model incorporating it from first principles, e.g. conservation laws or symmetries. However, subjectivity is present in this approach: How to check whether enough complexity is included? How to compare nearby models? How to ascertain the structural stability of the description, i.e. its keeping its validity if more physics is included? Furthermore, very often implicit assumptions are made in the process. At each step of the complexification, new temporal and spatial scales may show up, as well as new dimensionless numbers. The analytical or numerical treatment of the model becomes also more tedious, and possibly its experimental check as well. Therefore, going to the more complex tends to be done with caution, and there is a trend toward extracting as much physics as possible from a potentially too rough model. Furthermore, because of the lack of knowledge about the conditions of validity of a model, one cannot be sure that a more complete set of equations of this model is bound to bring an improved description of the system of interest ${ }^{7}$. Finally, simpler models are easier to present to colleagues or to teach to students, and their results are easier to discuss. The quote attributed to Einstein "Make everything as simple as possible, but not simpler" states

\footnotetext{
6 A very interesting example where this process was applied repeatedly is the theory of edge localized modes (ELMs) in tokamaks. First they were considered as current driven, then as pressure driven, then back as current driven, and now as both pressure and current driven (see chapter 3 , section 2.6 .3 of [127], and [114]).

7 As was told in section II A 2, the applicability of MHD equations to fusion plasmas may be justified if perpendicular motion dominates, at least for the mass and momentum equations. Therefore adding more MHD equations to the latter does not mean necessarily a more accurate description. Furthermore the use of the mass conservation equation may bring an unphysical peaking of density on the axis of a pinch which is avoided by microscopic turbulence in an actual plasma (see section 9.3.3 of [17]).
} 
an aim that is beyond one's reach for complex systems ${ }^{8}$ !

The articulation of fragmentary descriptions into a global one often is a personal and implicit process driven by interactions with the scientific community. This process is fed by the intuition developed by the knowledge of many experimental facts and simple models. Since at any moment a researcher stands in between knowing everything about nothing and nothing about everything, his global view has fuzzy outskirts. So is that of his community. Tradition plays an important role, which reminds of law reasoning, and of historical, biological, and hereditary logics. Scientific schools are bound to develop in a "blind men and the elephant" process.

\section{Validation and refutation of assumptions}

It is well known that " $A \Rightarrow B$ " and " $B$ right" does not prove that $A$ be right. However, implicitly people often act as if $A$ were right. In physics, when a theory agrees very well with experimental data and makes right predictions, it is often considered as right ${ }^{9}$, possibly with precise ideas about its actual real range of validity (for instance for classical mechanics when thought in the context of quantum or relativistic mechanics). However " $A$ is right" may happen to be said even for a theory which has only partial agreement with facts or which cannot be precisely tested, because it is the only one available, or because it is simple, or elegant, and taught to students. Furthermore proving that $A$ is wrong may be difficult and may take years for a complex system, because of the lack of information about it.

The present theory of neoclassical transport is an example of reference theory which has not been tested, because it is hidden by turbulent transport which is ubiquitous in magnetized plasmas. Even for theories about anomalous transport, a clear-cut experimental check is difficult, in particular for two cases: (i) the existence of a residual stress in momentum transport [102]; (ii) the existence of fractional diffusion (see [35, 36] and references therein) challenging the standard advection-diffusion picture ${ }^{10}$. These two checks are very difficult, since the calculation of profiles of transport coefficients belongs in the category of inverse problems which are known to come with issues of ill-conditioning or singularity. Transport codes provide a classical way to infer the profile of transport coefficients in fusion plasmas: assuming given functionals for the profiles of transport coefficients, the free parameters are iteratively adjusted to best reproduce the measurements. However this does not provide any estimate on the uncertainty of the reconstructed profiles $^{11}$.

A case of simple and elegant theory with only a partial agreement with facts, is Taylor theory of magnetic relaxation [116] applied to the reversed field pinch ${ }^{12}$ (RFP). When it was published in 1974, this theory brought the first theoretical attempt to explain the mysterious RFP relaxation, and had several features in agreement with experiments. However, the theory was unable to explain the dynamo at work in the plasma and the features of its outer part. Furthermore it described a driven ohmic system as if it were closed. Nevertheless it became the reference model, as shown in particular by reference [101] which provides a list of shortcomings of this theory in its section 3.6 though. In 1999, new MHD numerical simulations gave momentum to the new paradigm of single helicity equilibria [26, 65], already introduced in 1990 [22, 23, 67]. This was an incentive for experimentalists to look into the data base of RFX, the largest RFP, and to find that quasi single helicity states had been present in many discharges for quite a long time. Taylor relaxation theory was not consistent with these states (see section 7 of [27]), which forced to give up this paradigm [55].

Another interesting case in the frame of fluid theories is the ability of the simplest Ohm's law to enable the correct calculation of the saturation of various resistive instabilities. Indeed with general assumptions, the supplementary terms of a generalized Ohm's law do not contribute into the parallel Ohm's law used in such calculations (see section

8 However plasma physicists are not desperate. To the contrary going ahead by using many approximate models is like going downhill rapidly on a scree made up with small stones: this is both fast and pleasant, though none of the stone be comfortable to stand quietly! However plasma physicists may sometimes be meditative: "(..) had the range of instabilities now known to beset tokamaks been discovered by theoreticians before the experimental program was undertaken, there might have been some hesitation" (p. 562 of [121])...

9 According to Popper's falsifiability paradigm, this theory just survives the process of refutation, but it is not protected from refutation in the future [105].

10 This picture is quite flexible though, and is justified for generic particle transport, provided there is enough randomness in the Hamiltonian describing the dynamics [60].

11 Some progress may be expected from a new technique tailored for periodically modulated experiments [61]. This technique avoids any a priori constraint on the profiles, and computes them by simply inverting a $2 \mathrm{D}$ matrix. It also provides the uncertainty on the reconstruction. This is done by a controllable smoothing of the experimental data, instead of the ad hoc regularization of the profile of transport coefficients operated by transport codes (see Appendix 3).

12 The RFP is a magnetic configuration germane to the tokamak, that produces most of its magnetic field by the currents flowing inside the plasma [55]. With respect to the tokamak and the stellarator, the RFP has a low imposed external field. It has a helical magnetic field like the stellarator, but it is more magnetically self-organized than a tokamak, and much more than a stellarator. 
2.1 of [25]). This does not prove the validity of the simplest Ohm's law. Indeed the plasma velocity may strongly depend on the Hall contribution for instance.

Many simple models recover Landau damping in an intuitive way. As stated in section III A, some of these models make wrong assumptions, though their prediction about the existence of the damping be right.

\section{Difficulty in being critical and in changing views}

A classical reference for the scientific method is provided by the four principal rules of Descartes'Discourse on the Method (1637). In rough words, one should apply a systematic doubt to any new statement, divide each of the difficulties under examination into as many parts as possible, go from the simplest to the more complex, and make enumerations so complete, and reviews so general, that one might be assured that nothing was omitted. As told before, each expert has his own global view about a given class of plasmas. In reality, he stands very far from the four Cartesian rules:

- He must provisionally accept for true assertions that are not clearly known to be such.

- The difficulties under examination have so many aspects that there is no way to find an adequate solution by dividing all these aspects.

- There is no way to ascend little by little to the knowledge of the more complex, by commencing with objects the simplest and easiest to know.

- As a result, there is no way to make enumerations so complete, and reviews so general, that one might be assured that nothing was omitted.

All this sounds quite deceptive, but the Cartesian dream is implicitly at work in the community working on a given class of plasmas, and induces a polarization of the heuristic efforts. However, at a given moment this community, though being made up of physicists who aim at being critical (Cartesian doubt), must accept to think in a way similar to the legal reasoning where rationality is continuity: "In view of the accumulated knowledge, it is natural to think that..." Therefore tradition plays an important role.

An explicative model tying together the past knowledge about a given phenomenon is forcedly accepted reservedly due to the above necessary practical violation of the Cartesian rules. Therefore, challenging new experimental or theoretical results may first be felt as a mere confirmation that the as yet accepted model is not perfect, but not as a proof it is breaking down. At some moment, a new explicative model may be considered, but still keeping in mind the former one: the glass is both half-full and half-empty. Giving up on past habits is difficult to justify, since there are so many facts and factors to account for in order to provide a good theoretical description. This makes all the more difficult the painstaking change of paradigm described by Kuhn [87], and first advocated by de Broglie ${ }^{13}$. This mental dynamics is present, for instance, in the evolution of the theoretical view about sawteeth, ELM's (see footnote 6), and disruptions in tokamaks [79]; also about the RFP, as is now described in two steps.

Till the 2000's, Taylor relaxation theory (TRT) was so strongly accepted that another explanation of the partial magnetic relaxation observed in the RFP, proposed in 1991, was overlooked, though very simple and closer to experimental facts, as far as the current distribution is concerned: the Rusbridge theory inspired by ideas of Kadomtsev and Moffatt ${ }^{14}[111]$

Though the existence of long-lasting quasi-single helicity states in RFX invalidating TRT was published in 2000 [64], it took almost a decade for the change of paradigm to become obvious with the cover story of Nature Physics in August 2009 stating: "Reversed-field pinch gets self-organized" [92]. Here, another interesting phenomenon occurred: when motivated by numerical simulations, experimentalists looked into the data base of RFX, and found that quasi single helicity states had been present in many discharges for years, but not analyzed because they were considered as atypical (out of the paradigm): in agreement with Kuhn's view [87], the previous paradigm led to a screening of the facts challenging it. It was a pity, because single helicity was predicted theoretically in 1990 (Cf. section II A 4), and comes with good magnetic surfaces, while multiple helicity comes with broad magnetic chaos.

\footnotetext{
13 "The history of science shows that the progress of science has constantly been hampered by the tyrannical influence of certain conceptions that finally come to be considered as dogma. For this reason, it is proper to submit periodically to a very searching examination principles that we have come to assume without discussion." [34]

14 The explanation goes along the following steps: (i) In the radial domain where the magnetic field is chaotic, transport is fast, and the equilibrium is almost force-free; therefore $\mathbf{J}=\mu \mathbf{B}$ where $\mu$ may be space-dependent. (ii) Setting this in $\nabla \cdot \mathbf{J}=0$, implies $\mathbf{B} \cdot \nabla \mu=0$, which shows that $\mu$ must be constant along field lines; thus $\mu$ is constant in the chaotic radial domain. This straightforward derivation yields a result in full agreement with the fact that, in MHD simulations, $\mu$ is almost constant in most of the domain with a positive toroidal magnetic field, but not where it is reversed [24]. However this domain of almost constant value of $\mu$ was rather considered as a hint to the validity of TRT which predicts $\mu$ constant over the whole plasma radius instead [116].
} 


\section{Numerical simulation: a complex tool to face complexity}

Numerical simulation is a tool that enables a dramatic progress in the description of complex systems. In the past, only analytical calculations were possible, which limited strongly the set of tractable models. This reinforced the "look under the street light" syndrome. For instance, the simplest MHD model to describe RFP relaxation is made up of Navier-Stokes equation including Laplace-Lorentz force, and Faraday-Ohm's law. In 1974, there was no way to simulate adequately this set of equations. As a result, the already mentioned Taylor relaxation theory [116] was built with the Ansatz that fluid motion might be neglected, and that relaxation might be described in a purely magnetic way. Fifteen years later, good simulations were possible and revealed features of RFP relaxation that ruled out TRT [27]. Among them, the single helicity states were already mentioned, but the paramount importance of fluid motion was exhibited too. This motion explains the dynamo component of the electromotive force driving the currents in the plasma, which was a mystery for the previous TRT paradigm. At present, single helicity states are understood as a mere extension of the saturated tearing mode, in particular as far as the electric drift nature of the dynamo is concerned $[19,25]$. As yet, MHD simulations remain the main way to address theoretical issues, since the analytical description of these states is just in its infancy ${ }^{15}[18,25]$.

In many other fields of plasma physics too, numerical simulations are the irreplaceable tool for investigating complexity $^{16}$. This has made the issue of verification and validation of these codes a crucial one [118]. However, the role of intentionality is higher in simulations than in analytical calculations because of the choice of initial conditions and of parameters. The essentially dynamic nature of simulations mimics the experimental behavior of plasmas, but with a much higher representational ability than provided by measurements on the one hand, and with a much smaller complexity than actual experiments on the other hand. Though numerical experiments have an experimental character, they often come without error bars on their predictions, in particular when they involve an underlying chaotic dynamics. The numerical coding of an analytical model often involves many uncontrolled approximations. For instance the name "Vlasov code" encompasses very different types of codes, all with non Vlasovian features. Eulerian Vlasov solvers produce a fake diffusion in phase space that violates Liouville theorem. Particle-in-cell codes in reality simulate a dynamics which is more Klimontovich-like than Vlasovian. They avoid fake diffusion at the expense of a higher noise because of a much smaller plasma parameter $n \lambda_{\mathrm{D}}^{3}$ than in real plasmas. However both approaches have the advantage of avoiding the development of unphysical very thin Vlasovian filaments in phase space.

As told above, complexity may be added to a simple model in many different ways and this leads to the parallel use of codes with various abilities. However it would be very useful to check a posteriori what are the dominant terms in the calculation. As to nonlinear terms, which ones act only through their linear contribution (especially for a final "stationary" state). This might be a lead for the development of, possibly analytic, reduced models.

A present trend in magnetic fusion research is to develop an integrated modeling of tokamaks by tying together a bundle of codes. This will certainly bring a new knowledge of complexity, but new techniques will be needed to check the validity of the integrated models.

Despite the growing importance of numerical simulations, analytical calculations of simple models have remained important and will stay as a reference. Indeed, an analytical calculation reveals the internal structure of a model, its dominant parameters, it has an intrinsic flexibility with respect to the parameters values, and it can be checked more easily than a numerical simulation. Analytical calculations can be used to verify numerical simulations and avoid some of their pitfalls: cancelations of large terms, problems of stability and convergence due to insufficient numerical analysis, fake boundary effects, fake dissipation, etc... Fortunately, computers also help for such calculations with computer-assisted algebra. Sometimes numerical simulations can suggest assumptions for a new analytical approach (an example is given in section III E).

A final caveat might be useful. Indeed the growing power of computers, and the progress in numerical analysis and data processing make numerical simulations increasingly powerful... and fascinating to students. However numerical experiments are not true experiments. Only the latter are the ultimate beacon for understanding physics. Therefore students should be encouraged to become experimentalists. This point is dealt with in section II B $9 \mathrm{~b}$.

15 However this description already reveals that the edge current does not matter to reach shallow reversal. This is important to guide the endeavor toward improving confinement of quasi-single helicity states: one should enable the central part of the plasma to reach a genuine ohmic equilibrium. Indeed this should induce a low resistivity central part of the discharge diminishing the loop voltage, and thus the ohmic power for the same plasma current.

16 It is hard for young physicists to imagine the age where numerical simulations were a tour de force with card punching, batch submission, and paper outputs. In 1976 the author published a one-dimensional Vlasovian simulation with 8000 cells in phase space, while in present codes this number is larger by more than three orders of magnitude! However this lean code enabled to uncover the thermalization of a volume-created plasma due to the lack of static equilibrium [51]. 


\section{B. Possible methodological improvements}

We have just described the empirical way plasma physicists deal with complexity. This section considers how this description might be improved in view of the difficulties presently faced by plasma physicists. One of these difficulties, information retrieval, is specific to plasma physics and possibly more generally to the physics of complex systems. Other ones, as the inflation of publications and the growing importance of oriented programs, are a general problem of contemporary physics. Working on complex systems is a hard task, but the present trend of scientific practice makes it even harder. Therefore plasma physicists would gain very much in any improvement of this practice, and might be motivated into impelling a change. Challenges like ITER and DEMO might benefit a lot from such an improvement.

Here we venture some proposals for improving the present situation along the following lines : improving the way papers are structured and the way scientific quality is assessed in the referral process, developing new data bases, stimulating the scientific discussion of published results, diversifying the way results are made available, assessing quality more than quantity, making available an incompressible time for creative thinking and non purpose-oriented research. Some possible improvements for teaching are also indicated.

\section{Claim section}

Many improvements may be thought of to make plasma research easier. Some of them would mean an evolution of the rules of various research organizations, which is hard to trigger on large scale in the plasma physics community. However, there is a direction which might be improved rapidly and on an experimental basis: the structure of scientific papers, in order to avoid the "can't see the forest for the trees" syndrome. Indeed, it is often difficult to get the point of a given paper from its abstract, introduction and conclusion, in particular to find out what are the most important figures or formulas. A part of the problem comes from the haste to publish new results, which does not help in their pedagogical presentation. This also leads to misunderstandings from referees, which increases the number of referral runs. In the latter process, the paper may be clarified, but possibly only in a marginal way, which leaves its understanding still difficult to future readers.

A corrective action could be each research paper, even letters, to have a claim section being a kind of executive summary, but without any stylistic effort, more a list of very synthetic claims. This section would first list the main results and then their most relevant connection to previous literature. This would provide a clear information to assess the importance, the originality, the actual scientific contribution of the paper, and about the "precedents, sources, and context of the reported work", as worded in the APS guidelines for professional conduct [125]. Salient figures or formulas would be set there to make the claims explicit. Therefore the claim section would be a lot more informative than a traditional abstract. The present chapter provides very few new scientific results, and thus a claim section would make little sense. Therefore two examples of claim section are proposed in Appendix 2 for paper [58] mentioned in section IIID, and in Appendix 3 for paper [61] mentioned in section II A 4.

It is worth noting that the very compact way of communicating new results of such a claim section is reminiscent of the way physicists communicate the essence of their results to their colleagues in the corridor of large congresses. It is also what each reader tries to do by himself when trying to get the essence of a paper without reading it entirely. This procedure is standard in the mathematical literature. It would also improve the clarity of the papers. Writing the claim section would lead an author to state the essence of his results in a more accessible way ${ }^{17}$, and without having to care about the literary constraints of a normal text. He would be led into a better assessment of the actual novelty of his work, which would be bound to improve both the abstract and the remainder of the manuscript.

\section{Improving the referral process}

When available, the list of claims could seriously help in assessing a paper. Indeed one might think the referee to be then required to assess each of these claims (right or wrong?) and to check the list of precedents, sources, and context of the reported work, before writing down the remaining of his referee report, or before filling in the report form. When choosing a "false" for a given claim, he should motivate his statement.

17 It helped for the present chapter, even under the disguise of an extended summary! The reader is invited to write a claim section for his/her next paper, in order to ascertain the interest of the method, even for a private use. This section might be put as an appendix in the paper. 
This procedure should make the referral process more scientifically rigorous and faster. Referees would be sure not to miss the importance, the originality, or the actual scientific contribution of the first version of a manuscript, as claimed by the author. They could better help authors to adjust their initial view about this. Therefore the claim section would decrease the number of referral runs, and would speed up the refereeing process. This would help referees into an ethical refereeing. Editors would benefit from a better refereeing process, which would avoid many author's complaints, while making faster the editorial process. They would also have more factual elements to assess manuscripts and referees. Journals would benefit from the increased clarity of the contents of their published papers. The procedure might start with an experimental stage where the claim section would be optional for the authors, but not for the referee report if the claim section is available.

\section{New data retrieval technique}

Finding the relevant information about a given topic in plasma physics is often a hard task. Empirically people use a mix of word of mouth, browsing textbooks and review papers, web research engines, and bibliographic databases. In reality, the capability of modern computers as far as data processing, databases, and hyperlinking are concerned might provide much better tools than those already available. An important direction of improvement might be structuring and articulating the knowledge about a given topic, especially when many papers deal with it, and when it has been developing for decades. Presently web research engines and bibliographic databases give so many papers, that finding the most relevant ones becomes a formidable task. In particular following quotations forward or backward in time provides an exponentially growing set of papers whose majority is likely not to be informative for the topic of interest. Indeed papers may be quoted for purely technical reasons, to illustrate side comments, because they are wrong, etc...

The claim sections might provide a simple way of developing a new technique for data retrieval adapted to plasma complexity. Indeed, for each paper this section might be set by the corresponding scientific journals or publisher into a new dedicated data base accessible through Internet where cross-referenced papers would be hyperlinked. Their hyperlinking would provide a collaboration between scientific journals and publishers, but an implicit one, requiring only marginal legal agreement. It would facilitate the assessment of the state of the art on a given topic, with respect to what is available through present bibliographical databases. Indeed, connectivity between the various papers would be more topic oriented. APS guidelines for professional conduct [125] state that "It is the responsibility of authors to have surveyed prior work in the area and to include relevant references." Presently it may be hard, even with good will, to fulfill this requirement. This might become a lot easier with the new data base.

The spontaneous development of these data bases would probably only incorporate new papers and old ones that are still known, but some relevant old ones might be overlooked. After the start-up phase of the bases be over, further work would be necessary to screen the past unquoted literature. Public money could be involved in this second stage, since publicizing old results is a way to save present and future research work.

\section{Stimulating the scientific discussion of published results}

With the claim sections and the corresponding hyperlinked database, researchers might be made more responsible about the contents of their papers. A feedback system should be developed to this end. In reality, there is already one: the comments sent to a journal about its published papers. Unfortunately this system is very rigid and formal, and people sometimes feel its use as unfriendly. In order to cope with this issue, one might consider broadening the way papers are commented by adding a first, friendlier step: a researcher who would disagree with the contents of a paper would get in touch with its authors directly and try to sort out the issue. If they finally agree that there is something wrong, they would publish a common short corrective communication, naturally linked to the original claim section of the original paper. A classical comment to the journal would be sent only if the authors could not agree about a common view. It would also be useful for journals to enlarge this two-step comment system in order to enable comments about publications not present in journals, but in books, articles in proceedings, etc... This new kind of scientific discussion might be encouraged by the various scientific societies linked to plasma physics.

The claim sections, the corresponding hyperlinked database, and the new comment system should lead toward talks, courses, and papers that would be more updated. They should also help to have a better view of the importance of papers. Naturally these tools will be naturally complemented by blogs, wikis, discussions in social networks, and other electronic means which are developing. 


\section{5. $\quad$ Praising quality not quantity}

It is well-known that quality is not a consequence of quantity, but the number of publications has been gaining a growing importance in the last decades in assessing physicists and research teams. This drives inflation in the number of publications. As a result, unfortunately no physicist has the time to read the so many papers corresponding to his field. An even sadder fact is that this is not a serious issue for most of these papers! Indeed a large part of the published literature brings marginal improvements to the knowledge of a given scientific domain, which are of interest only to a small subgroup of experts. However, it is a serious issue for the most important papers which might be overlooked because they stand in a crowd. This brings the first issue: "What are the important papers?", especially when going out of one's specialty.

A collateral effect of the inflation of publications is the parallel one of the number of papers to referee. Another negative trend is the growing importance of oriented programs and of the corresponding assessment process. These two trends are strongly time-consuming. Together with the incentive to publish many papers they decrease the time to think creatively and to read.

This encourages the following failings: mental inertia, works that overlook previous results (even if the corresponding paper is quoted!), parallel physics projects without contact, absence of scientific debate, fashions and related lobbies, etc... Therefore it might be very useful to make the assessment of scientific quality really quality, and not quantity, oriented. The interlinking of claim sections might provide a way to find out better the actual importance of related works, and to diminish the role of quantity in quality assessments. The same trend could be induced by diversifying the ways results are made available, as explained now.

\section{Diversifying the ways results are made available}

One might think about diversifying the ways results are made available:

- Discussion papers might be sent to experts in the corresponding domain in order to have a chance to listen to criticism, to add possible relevant quotations, and to improve the contents before submitting a paper for publication.

- Preliminary works might be made available on databases like arXiv, and modified or canceled later.

- The wording of papers might be more problematic. Sometimes this might be reflected even in the title by making it interrogative, in particular for papers about debated issues.

- The use of scientific wikis might be generalized.

- Journals might systematically propose a whole hierarchy of papers including short letters about breakthroughs, short, long and review papers. Follow up papers might be allowed with a short format, in particular for their introduction, and explicitly indicated in journals as such... and in publication lists! Referees would be requested to detect such papers in their review process, even if the follow up paper is submitted to another journal than the original one. This issue becomes important with the development of numerical simulations. Indeed any modification in the simulated model, or in the simulation parameters brings virtually a new result: how important is it?

- Attempts that fail are numerous and time consuming. Natural ones are bound to be repeated by several researchers. Why not allowing the publication of short communications describing such unsuccessful attempts?

The issue of publication in books is a tough one. Indeed they are generally less accessible than journals. Writing a book is a strongly time-consuming task, but it may be little rewarding from the view-point of scientific communication... Finally the current trend of electronic publication sets the issue of its long term archiving. Two new directions in scientific communication are worth mentioning: the "Article of the Future" [129] and the "Quantiki" featuring 5 minutes presentations of new results in quantum mechanics [130].

\section{Time for creative thinking and non purpose-oriented research}

The idea of making available an incompressible time for creative thinking and non purpose-oriented research is now becoming popular with the "Slow science manifesto" [124]. Each researcher may try and do this, but it is important this to be recognized by scientific organizations too. More time for creative thinking, to look out of one's specialty, and to try transversal views may be a big saving for science ${ }^{18}$. A striking example comes from magnetic fusion physics. The tokamak is known to have a density limit which is proportional to the current density: the Greenwald density

18 This is the motto of the Institute for Advanced Study in Princeton [128]. 
limit $^{19}[75]$. Several papers have been published to provide tentative explanations of the phenomenon. Unfortunately, to the best of the author's knowledge, none of these theories works for the RFP where the same limit is present $[106,107]$, but this has been overlooked by tokamak experts.

It would be very useful to have a transversal view on magnetic confinement by using the information available from various configurations for magnetic confinement. Indeed important physics issues need to be solved both for ITER and for the definition of future demonstration reactors: what is the origin of the Greenwald density limit, how do transport barriers form and stay ${ }^{20}$, what is the origin of plasma rotation, what is the effect of additional heating, how to scale reactor parameters out of smaller experiments, how dangerous fast particle driven MHD modes may be, what is the benefit of a helical deformation of the magnetic field, what is the role of ambipolar electric fields, etc..? In particular, understanding the density limit in magnetic confinement might enable to come closer to this limit or to overcome it, and would increase considerably the reactivity of thermonuclear plasmas, which would dramatically increase the prospects of magnetic fusion. It is probable that important progress in this direction might be done by taking advantage that this limit is the same Greenwald limit in the tokamak and in the RFP, as said before.

Another topic where a transversal view would be useful is the dynamo. Indeed since (half) a dynamo is acting in the RFP, there is a natural resonance with the astrophysical dynamos. The corresponding communities have been interacting for several years, in particular in the frame of the Center for Magnetic Self-Organization in the United States. The von Karman Sodium (VKS) experiment in Cadarache came with a striking result: an incompressible fluid dynamo can drive an RFP magnetic state all by itself ${ }^{21}$ ! It is striking that the incompressible turbulent flow produced by impellers leads to the same magnetic equilibrium as in a current driven pinch whose plasma is compressible. Understanding the universality of the RFP configuration might lead to a large leap forward of dynamo theory.

\section{Improving heuristics}

The claim sections, the corresponding hyperlinked database, and the new comment system would help into improving heuristics. Indeed researchers would have a simpler and more global view of their research field. Theoreticians would be incited to make more explicit the scientific contents of their calculations, to go to the essence of phenomena, and to look for universal features and applications. "Islands of knowledge" would have a tendency toward connection. Simple models would be urged into embedding in a broader physics context and checking their structural stability when going toward more complete descriptions. It would be easier for experimentalists to be aware of theoretical results and to challenge them, since these results would not be obscured by their technical surroundings. Similarly theoreticians would get an easier access to experimental results and would have more opportunities to think about, and to suggest new experiments. This increased interaction between theory and experiments would certainly enhance creativity, and improve the quality of papers. The easier way to follow connection between papers would also help people to look out of their specialty, and better feed their intuition.

\section{Improving teaching}

Plasma physics has plenty of facets, but it is important to have a global view hereof. This has to be addressed when teaching it. Knowledge in general, and all the more that on complexity, has a multi-scale structure. Therefore, teaching should introduce from the outset the concept of complexity, and try to exhibit the various scales of the structure. A caveat: analytical calculations are a powerful heuristic tool, but often make very slow the introduction of concepts. It is thus advisable to limit the cases where they are described in details.

In reality there is no single way to define the multi-scale structure of a given complexity. As a result, topics which are presented in a separated way for a given choice of the structure, are linked when choosing another view. The presentation of these links may be very useful, especially for graduate students.

19 This limit is an edge density limit above which the discharge cannot be sustained.

20 From this point of view one might again take advantage of the analogy of the RFP with the tokamak, since in the RFP such barriers are related to shear reversal too [74].

21 This experiment studies dynamo action in the flow generated inside a cylinder filled with liquid sodium by the rotation of coaxial soft-iron impellers (von Karman geometry). It evidenced the self-generation of a stationary dynamo when the impellers do not rotate with the same angular velocity $[72,96]$. The magnetic field averaged over a long enough time corresponds to a RFP magnetic state with a large $m=0$ mode (see Fig. 7 of [96]). 
a. Limited capabilities of models Since our models are generally imperfect, when teaching one should use them in a different way than in more axiomatic parts in physics. Students should be made conscious about the limited capabilities of models, especially at a graduate level. Here are a few examples.

If students are taught resistive MHD, they should be made aware that other dissipations than resistivity may be present in the plasma like viscosity or heat diffusivity; therefore the Lundquist number is just one dimensionless number among many other ones, and its importance may be challenged by other such numbers ${ }^{22}$.

In the last decade a series of analytical calculations computed the width of the magnetic island of a saturated tearing mode [2, 3, 59, 76, 94, 95], which revealed in particular the mechanism of the saturation [59]. However, when teaching students, one should make them aware that present calculations of the saturation solve the magnetic part of the problem, but that the fluid motion part is still unsolved. This is all the more important that this fluid motion is a simple example of a dynamo, i.e. the production of an electromotive force from a fluid motion [25]. The RFP single helicity equilibrium provides another instance which is an extension of the simplest case. There, numerical MHD simulations are necessary to provide a description of the magnetic part of the problem too. In particular, they reveal the essential role of resistivity in the nature of the equilibrium, showing that it cannot be deduced from the original closed system picture originally present in Taylor relaxation theory [116].

b. Teaching physics or calculations? When teaching, one might think about what researchers often do when looking at a theoretical paper in order to get the essence of the physical result: they skip the calculations, and possibly go to them later (normally they should check everything, but they cannot!). So do the mathematicians who separate the statement of their theorems from their proofs. It is natural for textbooks to provide the calculations related to each phenomenon, but is it necessary to systematically present them when teaching? Here are a few examples.

For instance, the description of many waves in plasmas goes through similar steps: linearization about an equilibrium and Fourier transforms in space and time. Such a calculation may be done once for a simple case, but then avoided for other types of waves. Then possibly some indication may be given about the clever way to go through the set of equations, in particular as far as the physics behind approximations is concerned. This would leave more time to discuss the physics of the wave.

This is all the more true that the Fourier decomposition sometimes hides the nature of the physics underlying the wave. For instance the plasma frequency $\omega_{\mathrm{p}}$ is naturally introduced as that of a harmonic oscillator corresponding to the vibration of an electron slab with respect to its neutralizing ion slab. Langmuir waves are then understood by placing side by side such slabs where nearby ones have electrons in phase opposition ${ }^{23}$. Similarly, drift waves can be intuitively understood as the juxtaposition of the drift bumps described in section II A of [80].

When teaching Landau damping, it is important to convey the physics behind it, in particular the fact that the damping of a Langmuir wave is due to a phase mixing of its constituting beam modes (Van Kampen modes), and that these modes stay during the damping, as shown by the wave echo effect. The synchronization of particles with the wave brings the physical mechanism unifying Landau damping and growth (see section III A). To the contrary one may wonder whether it is really important to teach the Landau calculation, especially when accounting that this calculation is not amenable to any intuitive interpretation ${ }^{24}$. Teaching Landau's calculation makes sense as a second stage of the introduction to Landau damping for students who are meant to become theoreticians.

As a result, it may be important to teach certain phenomena in a way very different from their initial derivation. Because of the limited capabilities of models, the status of analytical calculations is different from more axiomatic fields of physics. Heuristically, they are a powerful tool to uncover new hidden physics, but pedagogically one may avoid them to start with, and keep the physical ideas and the corresponding images ${ }^{25}$. Another incentive to diminish

22 In the fluid description of screw pinches, a classical model is provided by the combination of Faraday-Ohm's law and of Navier-Stokes equation with Lorentz force. Then the Lundquist number is an obvious parameter. One may refine this description by adding a heat transport equation, which provides a self-consistent definition of the temperature profile, and accordingly of the resistivity profile. Then the Lundquist number is no longer a parameter, but an output of the model.

23 See for instance section 14.2.1 of [54]. This sheds also a new light on the hydrodynamic or cold beam-plasma instability (section 14.3 .1 of [54]). Indeed a modulation with wave number $k$ of the beam density generates a forcing of the plasma at pulsation $\omega=k u$, where $u$ is the beam velocity, which feeds back on the beam density modulation. The response of a harmonic oscillator scales like $\left(\omega_{\mathrm{p}}^{2}-\omega^{2}\right)^{-1}$. For $\omega \gg \omega_{\mathrm{p}}$ the electrons react weakly due to their inertia, which rules out a positive feedback for such $\omega$ 's, and by continuity for $\omega>\omega_{\mathrm{p}}$. Then the plasma behaves like a classical dielectric, which screens the perturbing charge. As a result the unstable forcing must correspond to $\omega=k u \leq \omega_{\mathrm{p}}$ with a maximum for equality. This contrasts with the classical "negative energy" picture which rather suggests $\omega=-\omega_{\mathrm{b}}+k u$, where $\omega_{\mathrm{b}}$ is the plasma frequency of the beam, and does not tell why the instability occurs rather for $k u \leq \omega_{\mathrm{p}}$, and why it is the strongest for $k u \simeq \omega_{\mathrm{p}}$. This forced harmonic oscillator picture works also for other reactive instabilities.

24 Moreover this calculation gives no clue to the plasma behavior in the actual nonlinear regime where damping is a manifestation of stability of an infinite dimensional Hamiltonian system (see section III A).

25 Using the blackboard is an efficient way to avoid "runaway lectures", especially when calculations are presented. 
the amount of taught analytical derivations is the growing importance of numerical simulations to uncover new hidden aspects of the complexity of plasmas: their results should be taught too.

Teaching physics requires teaching experimental facts. If less time is dedicated to calculations ${ }^{26}$, more time may be devoted to experimental results and to their error bars. This may be done in various ways. In particular, students' attention is captured when the historical path leading to the present view about a given phenomenon is described. Generally this path includes iterates of the interaction between experimental and theoretical results. This exhibits the fascinating character of the scientific adventure, and shows how important are experiments ${ }^{27}$... and experimentalists! This provides a balance to the fascination of computer work already mentioned in section II A 6 .

c. Taking advantage of nonlinear dynamics and chaos Plasma physics developed in a progressive way, and often textbooks are more the accretion of successive layers of knowledge than a presentation of its global reconstruction. In particular, nonlinear dynamics and chaos might provide a way to revisit and unify separated chapters ${ }^{28}$, e.g. turbulent and collisional transport, the calculation of magnetic field lines ${ }^{29}$, or the introduction of fluid and of Vlasov equations. The statistical mechanics of systems with long range interactions may bring useful complementary... and surprising views $^{30}[21,32]$. So does the theory of fluid turbulence ${ }^{31}$.

In particular it would be interesting to revisit with modern nonlinear dynamics and chaos what is usually called collisional transport in plasmas. The simplest instance of this transport deals with a uniform non magnetized plasma. It is traditionally described by considering the motion of a test particle due to the Coulomb force of particles within the Debye sphere (radius $\lambda_{\mathrm{D}}$ ) around it. Within this sphere two scales are important: the typical interparticle distance $d=n^{-1 / 3}$ and the classical distance of minimum approach $\lambda_{\text {ma }}=e^{2} /\left(4 \pi \epsilon_{0} k_{\mathrm{B}} T\right)$ where $\epsilon_{0}$ is the vacuum permittivity, $k_{\mathrm{B}}$ is the Boltzmann constant, $T$ is the temperature, $n$ is the density, and $e$ is the electron charge. These scales verify $\lambda_{\mathrm{D}} \gg d \gg \lambda_{\mathrm{ma}}$. The particles away from the test particles at a distance much larger than $d$ are not felt individually, but act through their mean field. To the contrary, a particle at a distance much smaller than $d$ is felt individually by the test particle and its Coulomb field dominates over that of all other particles. It is then natural to think of the interaction between these two particles as a two-body Rutherford collision. The effect of particles at a distance of the order of $d$ can be described neither by a mean-field description, nor by the two-body Rutherford picture: the test particle experiences Coulomb forces with the same order of magnitude due to several such particles, but not many.

Historically two groups at UC Berkeley's Radiation Laboratory derived at almost the same time a Fokker-Planck equation describing "collisions" in non magnetized plasmas and quoted each other results in their respective papers: one by Gasiorowicz, Neuman and Riddell [73] and a year later one by Rosenbluth, MacDonald and Judd [110]. The first group of authors dealt with the mean-field part of the interaction by using perturbation theory in electric field amplitude. The second group of authors used the Rutherford picture. Each theory has a difficulty in describing the scales of the order of $d$. The mean-field approach cannot describe the graininess of these scales, and the Rutherford picture cannot describe the simultaneous "collisions" with several particles. Even for scales smaller than $d$, the Rutherford collision is modified due to the fluctuating electric field of the other particles in the Debye sphere [73]. Using the more relevant description for scales smaller than $d[110]$ and the one for the larger scales [73], the corresponding contributions to transport turn out to be of the same order of magnitude. Furthermore, if one accepts to cross the "validity border" $d$, and one performs the final integration on the whole range of scales $\left[\lambda_{\text {ma }}, \lambda_{\mathrm{D}}\right]$ for either theory, the two results are found to agree [73, 110].

Because of gas dynamics, plasma physicists were led to think of the interactions of particles in kinetic unmagnetized plasmas within a Debye sphere as collisions. However, even though the Rosenbluth et al. paper provides the same result as Gasiorowicz et al.'s when both are applied to all scales within the Debye sphere, the Rutherford collision image is only correct for scales much smaller than $d$. Rigorously speaking one should not speak about collisional transport, but about "short range induced transport", "unscreened Coulomb interaction induced transport", or so.

The Gasiorowicz et al. approach has the merit to make a calculation of transport coefficients starting with the

26 Naturally this must be done without going up to a superficial presentation of the phenomena. Calculations are a way to anchor memory and to train students, especially at an undergraduate level.

27 In particular the development of new diagnostics to touch other parts of the "elephant".

28 This is all the more justified, since plasma physicists contributed a lot to the development of these topics.

29 Unfortunately, the beauty and the flexibility of the derivation of the Hamiltonian description of magnetic field lines by a stationary action principle [29] has been largely overlooked. It was formulated in a simple way in [104] and reference [41] showed a corresponding equivalence of canonical transformations and of changes of gauge.

30 For instance the existence of negative specific heat in a magnetically self-confined plasma torus [84]. The saturation of the cold and water-bag beam-plasma instability can be computed analytically by using Hamiltonian (1) introduced in section III with a single wave (cold: [70], water-bag: [8, 9]). The mean-field derivation of Vlasov equation was already mentioned in section II A 2.

31 There are strong analogies with plasma turbulence, as exemplified by the Charney-Hasegawa-Mima model, but also strong differences since plasma turbulence is seldom fully developed. Furthermore the word "intermittency" is used with quite different meanings in the two fields. 
genuine $N$-body dynamics using explicit assumptions and avoiding the ad hoc truncation of integrals at the Debye length. However, within the same approximations, a more elegant derivation of the same Fokker-Planck equation describing "collisions" in non magnetized plasmas is provided by taking the limit "infinite number of particles in the Debye sphere" of the Balescu-Lenard equation (see section 8.4 of [7] and sections 7.3 and 7.4 of [78]). With a single calculation, this derivation provides both the dynamic friction and the diffusion coefficient. As Gasiorowicz et al.'s approach, it also avoids the ad hoc truncation of integrals at the Debye length. It requires the plasma to be stable, which is a serious caveat for the applicability of the traditional Fokker-Planck equation to magnetized plasmas which are cluttered with instabilities ${ }^{32}$.

However the Balescu-Lenard approach still has an intrinsic shortcoming. Indeed, due to short range interactions, particle dynamics is chaotic in reality (this is implicit in Rosenbluth et al.'s theory), and one is facing the calculation of transport coefficients for a chaotic motion. The Balescu-Lenard approach makes a perturbation calculation which is not a priori justified for chaotic dynamics, even for scales larger than $d$. Therefore, students should be warned to be cautious, since, for the motion of a charged particle in a spectrum of longitudinal waves, a perturbation calculation yields the quasilinear estimate for the diffusion coefficient, while a super quasilinear regime, a synergetic effect in chaos, is found to exist in this chaotic dynamics for intermediate resonance overlap (see section IIID). There diffusion becomes quasilinear for strong resonance overlap, but not because the perturbation calculation becomes valid again (see section IIID).

As a result, as yet there is no correct calculation of the contribution of scales about $d$ to short range induced transport ("collisional transport"). This issue would be worth more theoretical investigation: how good are the classical and neo-classical theories of transport? This suggests plasma physics courses to have a part devoted to dynamics with the successive introduction of Hamiltonian chaos, of the transport due to short range interactions ("collisions"), and of turbulent transport. When dealing with "collisions", one might start with the true chaotic dynamics, and exhibit the different nature of the interaction for the scales smaller and larger than $d$. Then one could introduce the corresponding approaches with appropriate caveats: (i) the approximate perturbative approach à la Balescu-Lenard, with a recall of Gasiorowicz et al.'s work; (ii) the two-body approach of Rosenbluth et al.. Finally one might point out that the matching of the two theories is still an open issue: as yet only two parts of the "elephant" have been touched.

\section{DESCRIBING PLASMA DYNAMICS WITH FINITE DIMENSIONAL HAMILTONIAN SYSTEMS}

The main results of this section are summarized in the last three paragraphs of the extended summary in Appendix 1.

Due to its late development, plasma physics did not try to address its problems by a first principle approach, but borrowed many concepts and tools from other fields of physics like the kinetic theory of gases and fluid mechanics. In particular, in order to deal with kinetic aspects, people naturally looked for a description in terms of a velocity distribution function, and therefore for some substitute of the Boltzmann equation, and this was the Vlasov equation. This equation was the starting point of most of the kinetic treatments of plasmas, and the Vlasovian description is a must of any plasma textbook. However, as recalled in section II A 2, this equation is not justified for many time-scales where it is used, and some of the calculations it enables to do are far from intuitive (ex.: Landau damping).

However, in a system where the transport due to short range interactions ("collisions") is weak, it is natural to think about plasma dynamics by working directly with classical mechanics, and taking into account that the collective field dominates over the graininess field. Though natural, this did not occur spontaneously before the Vlasovian approach ran into a major difficulty: the description of the nonlinear evolution of the weak warm beam-plasma instability, or bump-on-tail instability. In the following, we only consider a one-dimensional plasma with electrons moving in a neutralizing uniform ion background ${ }^{33}$.

At the time where the Vlasovian approach ran into the difficulty of describing the nonlinear regime of the bump-ontail instability, the theory of chaos for finite number of degrees of freedom Hamiltonian systems had been developing in the plasma physics community for more than a decade, and this was an incentive to tackle the weak warm beamplasma instability by generalizing [53, 117] a model originally introduced for the numerical simulation of the cold beam-plasma instability $[98,100]$. There the beam was described as a set of particles while the wave was present as a harmonic oscillator. A Langmuir wave with a phase velocity $\omega / k$ where there are no resonant particles, as shown

32 The impact of instabilities was recently addressed in $[4,5]$ by taking into account the spontaneous emission of waves by particles which induces a corresponding drag on top of the "collisional" one.

33 This is a tremendous simplification with respect to the physics of many actual plasmas. In particular density fluctuations may bring dramatic changes in the dynamics of Langmuir waves by Anderson localization [37, 57], by a transfer of particle momentum over an increased range of velocities [50], and by nonlinear decay and scattering processes [123] 


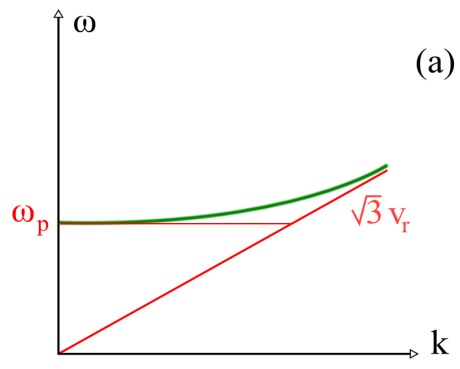

(a)

(b)

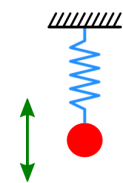
$\mathrm{k}$

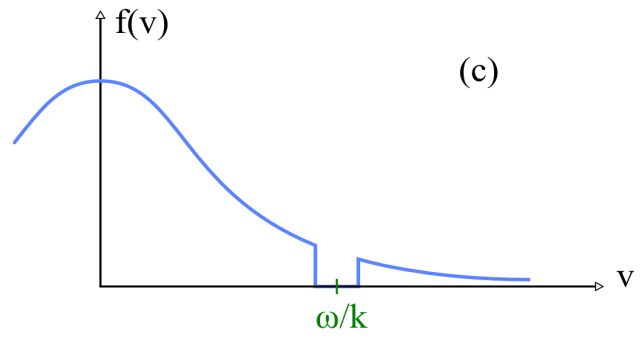

FIG. 1: Langmuir wave without resonant particles. (a) Bohm-Gross dispersion relation, (b) Equivalent harmonic oscillator, (c) Electron velocity distribution function with a gap at the wave velocity.

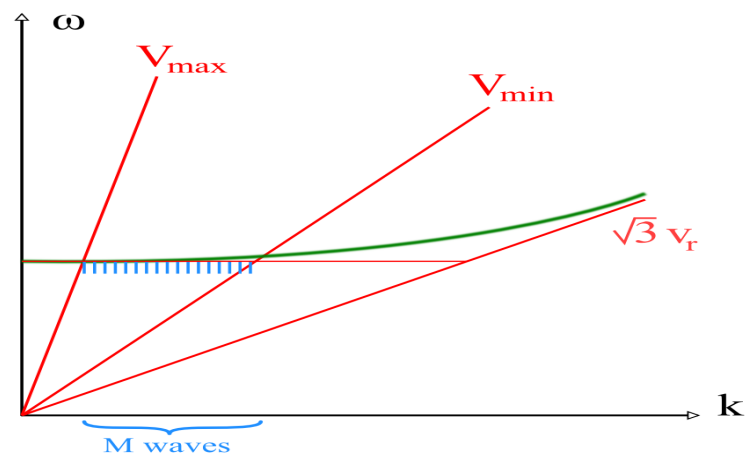

FIG. 2: Diagram showing Bohm-Gross dispersion relation with the velocity interval $v_{\min }, v_{\max }$ and a comb corresponding to the $M$ waves with phase velocities in this interval.

in figure $1(\mathrm{c})$, verifies the Bohm-Gross dispersion relation ${ }^{34}$ shown in figure $1(\mathrm{a})$, and is equivalent to a harmonic oscillator (figure $1(\mathrm{~b})$ ). If one considers a wave-particle interaction occurring in a finite range of velocities $\left[v_{\min }, v_{\max }\right]$, then it is sufficient to include in the Hamiltonian the waves with phase velocities in this interval, which defines their number $M$ (figure 2). This finally yields the self-consistent Hamiltonian

$$
\begin{aligned}
H_{\mathrm{sc}}= & \sum_{r=1}^{N} \frac{p_{r}^{2}}{2}+\sum_{j=1}^{M} \omega_{j 0} I_{j} \\
& -\varepsilon \sum_{r=1}^{N} \sum_{j=1}^{M} k_{j}^{-1} \beta_{j} \sqrt{2 I_{j}} \cos \left(k_{j} x_{r}-\theta_{j}\right)
\end{aligned}
$$

34 This relation makes sense, since we consider low amplitude waves with phase velocities much above the thermal speed. If these conditions are not satisfied, the issue is a lot more involved [11-13]. 


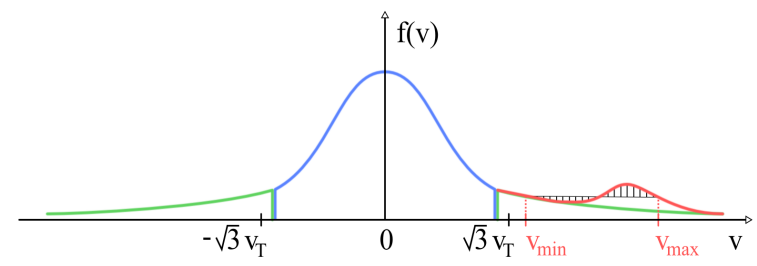

FIG. 3: Velocity distribution cut in three pieces: a non-resonant central part in blue, and left and right resonant parts in green for the case of a thermal plasma and in red for that of a bump-on-tail; the corresponding plateau in shown in black.

where $\varepsilon=\omega_{\mathrm{p}}[2 m \eta / N]^{1 / 2}$ is the coupling parameter and $\beta_{j}=\left[\partial \epsilon_{\mathrm{d}}\left(k_{j}, \omega_{j 0}\right) / \partial \omega\right]^{-1 / 2}$, with $\omega_{\mathrm{p}}$ the plasma frequency, $m$ the mass of particles, $\eta$ the ratio of the tail to the bulk density, $\epsilon_{\mathrm{d}}(k, \omega)$ the bulk dielectric function, $k_{j}$ and $\omega_{j 0}$ the wavenumber and pulsation of wave $j$. The conjugate variables for $H_{\mathrm{sc}}$ are $\left(p_{r}, x_{r}\right)$ for the particles and $\left(I_{j}, \theta_{j}\right)$ for the waves. On top of the total energy $E_{\mathrm{sc}}=H_{\mathrm{sc}}$, the total momentum $P_{\mathrm{sc}}=\sum_{r=1}^{N} p_{r}+\sum_{j=1}^{M} k_{j} I_{j}$ is conserved.

This model was derived from the $N$-body description of the beam-plasma system [1]. More recently, this was done again in a heuristic way (see section 2.1 of [48]), and in a rigorous one by a series of controlled approximations (see the remaining of chapter 2 of [48]), which enables replacing the many particles of the bulk by their collective vibrations. So, in figure 3 the blue central part of the distribution is no longer present as particle degrees of freedom; if one is interested in the evolution of the red bump, one may incorporate the left green wing into the bulk too. As shown in the next subsections, this approach helped into the investigation of the nonlinear evolution of the weak warm beam-plasma instability ${ }^{35}$. However its first contribution was to provide a rigorous mechanical understanding of Landau effect. It also provided a new insight into the transition from Landau damping to damping with trapping when the amplitude of a Langmuir wave is increased [99]: it turns out to be a second order phase transition [68], a phenomenon which is hidden in the full $N$-body description of the plasma when the same Gibbsian approach is used. It is worth noting that a self-consistent Hamiltonian description is also powerful for the description of wave-particle interaction for waves in magnetized plasmas for which the Larmor precession plays an important role (see [85] and references therein).

\section{A. Recovering Vlasovian linear theory with a mechanical understanding}

Before applying this model to the saturation of the beam-plasma instability, it was necessary to make sure that it included the physics of Vlasovian linear theory. Therefore, one had to address the linear theory of the perturbation of a spatially uniform velocity distribution function by small waves. In order to stay in the spirit of classical mechanics, this unperturbed state should correspond to a single mechanical system, and not to an ensemble of systems. This is naturally obtained by considering the unperturbed plasma as made up of a series of monokinetic beams, and each beam as an array of equidistributed particles. If the waves have a vanishing amplitude, this state is invariant in time. Then perturbation theory is performed by using mere Fourier series, and leads to a Floquet problem in $2(N+M)$ dimensions. In contrast with the simplest Floquet problem, the Mathieu equation, surprisingly this problem can be solved explicitly!

The solution includes the Landau instability [88] as an eigenmode if the distribution function has a positive slope. If the slope is negative, it does not provide Landau damping as an eigenmode, but only a series of beam modes. In agreement with van Kampen's theory [83], Landau damping is recovered as a result of the phase mixing of the latter. It must be stressed that in Hamiltonian mechanics, in agreement with time reversibility and with Liouville theorem, a damped eigenmode comes with an unstable one having the opposite exponentiation rate. Therefore Landau damping cannot be a damped eigenmode, since it would come together with an unstable one which would be seen with probability 1. Moreover, in Vlasovian theory, Landau damping is not an eigenmode, but a time-asymptotic damped solution obtained by analytic continuation. Furthermore, in Vlasovian theory it is not sure a priori that the van Kampen phase-mixing solution actually exists, and is not destroyed by nonlinear effects related to finite, though small, amplitude of the beam modes. Proving this nonlinear stability [97], and thereby the actual existence of

35 By taking advantage of the intuition developed by this approach it is possible to derive a more pedestrian approach to wave-particle interaction [54]. 
Landau damping, was a mathematical tour de force, the equivalent of a KAM theorem for continuous systems, and led Cédric Villani to be awarded the 2010 Fields medal. In the frame of the finite dimensional Hamiltonian approach, this nonlinear stability is the mere result of KAM theorem itself.

As André Samain pointed out, if the distribution function has a positive slope, an unstable and a damped eigenmode are not enough to recover the Vlasovian result. Indeed, a typical initial perturbation excites both modes with the same amplitude at $t=0$, but the damped one dies out, which leaves only the unstable one with half of the Vlasovian amplitude. In reality, a typical initial perturbation excites also a wealth of beam modes. When their contribution is properly taken into account, Yves Elskens found (section 3.8.3 of reference [48]) that an initial perturbation with amplitude 1 evolves in time according to the time-reversible expression $\mathrm{e}^{\gamma_{j \mathrm{~L}} t}+\mathrm{e}^{-\gamma_{j \mathrm{~L}} t}-\mathrm{e}^{-\gamma_{j \mathrm{~L}}|t|}$ : the beam modes act subtractively to compensate the damped eigenmode, and to restitute the Vlasovian solution. This apparent intricacy corresponds to experimental reality. If Langmuir waves are excited by a grid in a magnetized plasma column, this is done by the excitation of the various "monokinetic beams" going through the grid. Landau damping results from the phase mixing of these excitations which do not die out, as proved by echo experiments [6]. If a weak warm beam goes through the grid together with the background plasma, the beam modes are excited too and contribute to the Langmuir wave amplitude. As a result of this analysis, the Vlasovian limit, though very powerful, turns out to be a quite singular limit for the linear theory of waves.

At this point, we made sure the finite dimensional Hamiltonian approach recovers Vlasovian linear theory. However, the former approach comes with an important bonus: it brings the information of particle dynamics in parallel with the wave's. This is absent in the Vlasovian description and has two important consequences. First, because of its lack of intuitive contents, the reality of collisionless Landau damping was fully recognized only after its experimental observation in 1964 by Malmberg and Wharton [93], almost two decades after its prediction. Second, textbooks are forced to come up with complementary models to try and explain intuitively the way Landau effect works. The finite dimensional Hamiltonian approach enables to assess these models which are not all correct, unfortunately (see section 4.3 .1 of reference [48], in particular the exercise therein as a caveat ${ }^{36}$ ). Better, it shows Landau damping and instability to result from the same synchronization mechanism of particles with waves.

In order to avoid repeating here the whole argument, we give a simple proof that particles released at $t=0$ with a velocity $u$, and a uniform initial spatial distribution, have an average velocity which comes closer to the wave phase velocity over a bounded time. Let $\ddot{x}=\varepsilon \cos x$ be the equation of motion of the particle in the wave, expressed in the reference frame of the latter. Assume the unperturbed orbit to be $X_{0}(t)=x_{0}+u t$. A perturbation calculation to second order in $\varepsilon$ yields

$$
\Delta u(t)=\varepsilon^{2} \frac{\cos u t-1+\frac{1}{2} u t \sin (u t)}{u^{3}} .
$$

$u \Delta u(t)$ is even in $t$ and is negative from $t=0$ up to $t=T \equiv 2 \pi /|u|$, which means an average synchronization of the particles with the wave within this time interval whatever be the relative sign of their velocity to the wave. Since quantity $\Delta u(t)$ scales like $1 / u^{3}$, the average synchronization is small for large $|u|$ 's: it is a local effect in velocity. The effect is maximum for $|t| \simeq 3 T / 4$. For $t$ small, equation (2) becomes

$$
\Delta u(t) \equiv\langle\dot{x}(t)\rangle-u=-\frac{\varepsilon^{2} u t^{4}}{24},
$$

to fourth order in t. We notice that the effect vanishes for small $|u|$ 's. Therefore this effect is not related at all to trapping inside the wave troughs.

It can be intuitively understood as follows. Figure 4 displays a sketch of the phase space of particles moving in the presence of a wave. One particle is released at the position of the X-point of the separatrix (blue line), another one starting at the O-point of the trapping domain (red line). The first one has an orbit further away from the separatrix than the second one. Therefore it is less modulated, which provides their average synchronization over the considered time duration. This average synchronization effect was proved to exist in an experiment with a traveling wave tube [39].

Due to this synchronization, particles change their momentum. Since wave-particle momentum is conserved by the self-consistent dynamics, the wave changes its momentum, and thus its amplitude, in the opposite way. This brings the Landau effect [54].

The self-consistent calculation shows that the particles the most synchronized with the wave have a velocity about the growth rate $\gamma$ in the present units. For such particles time $\mathrm{T}$ is about $1 / \gamma$, the natural bound for the validity of a

\footnotetext{
36 In particular, though initially published with a caveat, the surfer model induces in the mind of the students the wrong feeling that
} trapping is involved in Landau effect. 


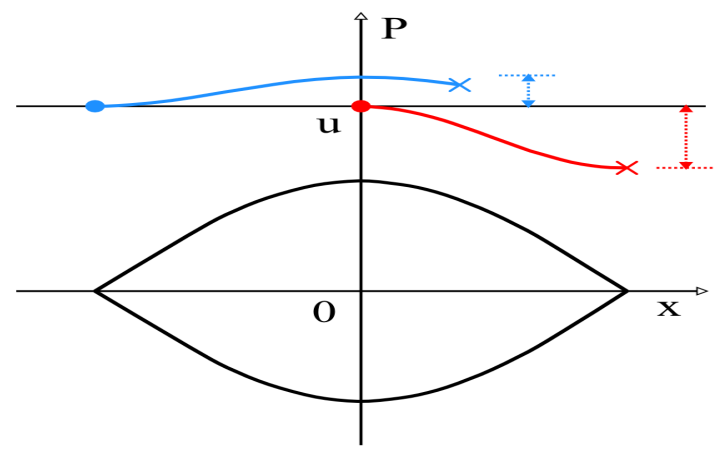

FIG. 4: Phase space plot displaying the average synchronization of two particles with a wave, one starting at the position of the X-point of the separatrix (blue line), another one starting at the O-point of the trapping domain (red line).

calculation with a wave of constant amplitude. The synchronization mechanism is the same for Landau damping and instability, which explains why the Landau effect is described by a single formula, though the physics of damping and instability display qualitative differences as far as the wave aspect is concerned. All this is hidden in the Vlasovian approach.

The Landau effect can also be recovered by a statistical approach ([63] and section 4 of reference [48]). There the wave phase and amplitude evolutions are computed by perturbation theory in the coupling parameter $\varepsilon$ of the self-consistent Hamiltonian. Together with the collective Landau effect, the calculation derives also the spontaneous emission of waves by particles. As a result, Landau damping turns out to be a relaxation mechanism driving waves to their thermal level.

\section{B. Quasilinear theory}

In 1961, Romanov and Filippov [109] introduced the quasilinear equations which were made popular in 1962 by two papers published in the same issue of Nuclear Fusion [40, 120]. As indicated by their name, these equations were derived by considering the nonlinear dynamics of the beam-plasma instability as close to linear, and more precisely by neglecting mode-mode coupling, except for its contribution to the evolution of the space-averaged velocity distribution function $\bar{f}(v, t)$. These equations are

$$
\begin{aligned}
\partial_{t} \bar{f} & =\partial_{v}\left(D_{\mathrm{QL}}(v, t) \partial_{v} \bar{f}\right), \\
\partial_{t} \psi & =2 \gamma_{\mathrm{L}}(v, t) \psi,
\end{aligned}
$$

where $\gamma_{\mathrm{L}}(v, t)=\frac{\pi}{2} \eta k^{-2} \partial_{v} \bar{f}(v, t)$ and $D_{\mathrm{QL}}(v, t)=\pi \eta \frac{1}{k^{2}} \psi(v, t)$ are the instantaneous Landau growth rate and QL diffusion coefficient, while $\psi(v, t)$ is the power spectrum, a smooth function going through points $\psi\left(t, v_{j}\right)=k_{j} I_{j}(t) /\left(N \Delta v_{j}\right)$ with $\Delta v_{j}$ the mismatch of phase velocity of wave $j$ with its two neighbors.

These equations show that at time $t$ an unstable Langmuir wave with phase velocity $v$ grows with the Landau growth rate $\gamma_{\mathrm{L}}(v, t)$ computed with $\bar{f}(v, t)$, and that the instability saturates due to the diffusion of the velocities of particles, which levels out the bump on the tail of the distribution function and substitutes it with a plateau (see figure 3). They also predict the wave spectrum at saturation which is shown in the upper part of figure 5 . Within experimental uncertainties, these predictions were confirmed by the first laboratory experiment looking at the bump-on-tail instability [108].

However, the perturbative approach used in the derivation of the quasilinear equations cannot be justified theoretically during the whole saturation of the instability. Indeed, waves scatter the particle positions with respect to their ballistic value. When the corresponding spreading of positions becomes on the order of the wavelength, the perturbative approach fails. The corresponding spreading time $\tau_{\text {spread }}$ turns out to be the (Lyapunov) time of separation of nearby orbits in the chaos induced by the waves, and also the typical trapping time of particles in the turbulent electrostatic potential. Obviously, leveling out the bump on the tail of the distribution function needs a time longer than the latter times. Therefore one might doubt at the validity of quasilinear equations to describe the saturation of the instability. In 1984, Laval and Pesme proposed a new Ansatz to substitute the quasilinear one, and predicted that whenever $\gamma_{\text {Landau }} \tau_{\text {spread }} \ll 1$ both the wave growth rate and the velocity diffusion coefficient should be renormalized by a factor 2.2 [89]. This motivated Tsunoda, Doveil, and Malmberg to perform a new experiment with a traveling 


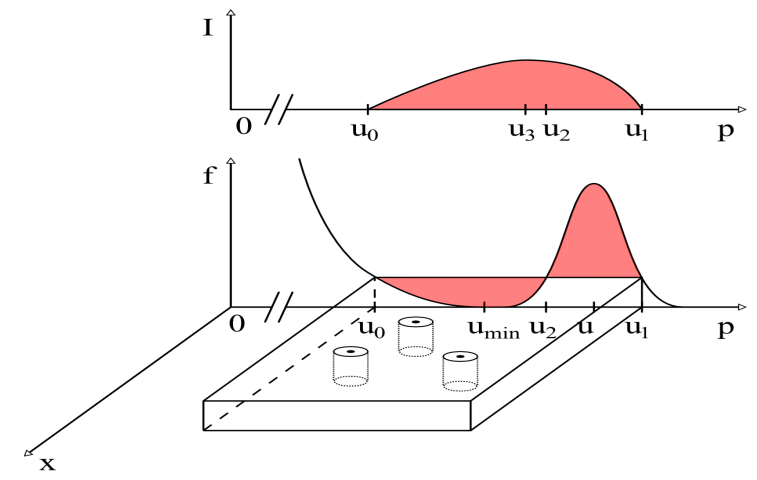

FIG. 5: Saturation of the bump-on-tail instability. Upper part: wave spectrum. Lower part: plateau both in velocity and in space. The vertical cylinders indicate groups of particles

wave tube in order to decrease the noise due to the previous use of a magnetized plasma column [119]. It came with a surprising result: quasilinear predictions looked right, while quasilinear assumptions were completely wrong. Indeed no renormalization was measured, but mode-mode coupling was not negligible at all. Apparently one had " $A \Rightarrow B$ " and " $B$ right", but $A$ wrong! This set the issue: would there be a rigorous way to derive the quasilinear equations ${ }^{37}$ ?

\section{Dynamics when the distribution is a plateau}

First, it is important to notice that one does not need the quasilinear equations to be correct to prove the formation of the plateau. Indeed this formation comes from the chaos induced by the unstable Langmuir waves among the resonant particles, whatever be the precise description of the corresponding chaotic transport. When the plateau forms in velocity, density becomes also almost uniform spatially in this range of velocities (see figure 5). Indeed, chaos tends at equidistributing particles all over the chaotic domain in phase-space. Actually, KAM tori, bounding the chaotic domain defined by a prescribed spectrum of waves, experience a sloshing motion due to the waves. This brings a small spatial modulation to the particle density which provides a source term for the Langmuir waves. However, if the plateau is broad, the evolution of the wave spectrum is slow, which brings only a small change to the previous simplistic picture of a uniform density (see section 2.2 of [15]). Therefore, there is almost no density fluctuation to drive the wave evolution as defined by the self-consistent dynamics: the wave spectrum is frozen. Hence the particle dynamics is the one defined by a prescribed spectrum of waves. Clumps of particles may experience a strong turbulent trapping, but the distribution function is unaffected by this granular effect. As a result, selfconsistency vanishes in the plateau regime if the plateau is broad enough, because particle transport only rearranges particles without changing the height of the distribution function itself within the plateau in phase-space, depriving waves from a source ${ }^{38}$ (the little cylinders in figure 5 keep their height while moving). This is an instance where nonlinear effects increase the symmetry of the system, and lead to a depletion of nonlinearity ${ }^{39}$.

This means that, when the plateau is formed, the diffusion coefficient $D(v)$ of particles with momentum $v$ is the one found for the dynamics of particles in a prescribed spectrum of Langmuir waves. Let $D_{\mathrm{QL}}(v)$ be the quasilinear value of this coefficient. The next section discusses the possible values of $D / D_{\mathrm{QL}}$ in the resonance overlap regime.

37 This academic issue has a broader relevance since the QL approximation is used everywhere in plasma physics.

${ }^{38}$ For a plateau with a finite width, the small remaining source brings a further evolution of the wave-particle system toward a Gibbsian state where the wave spectrum collapses toward small wavelengths together with the escape of initially resonant particles towards low bulk plasma thermal speeds [70]. This corresponds to a further step toward a new thermal equilibrium of the $N$-body system corresponding to the initial beam-plasma system. The description of the subsequent steps toward thermal equilibration require to use a full $N$-body model.

39 This phenomenon, also called depression of nonlinearity was introduced in fluid mechanics [86], and was identified as a result of the emergence of long-lived vortices where the enstrophy cascade is inhibited. It also exists in systems with quadratic nonlinearities [20, 86]. 


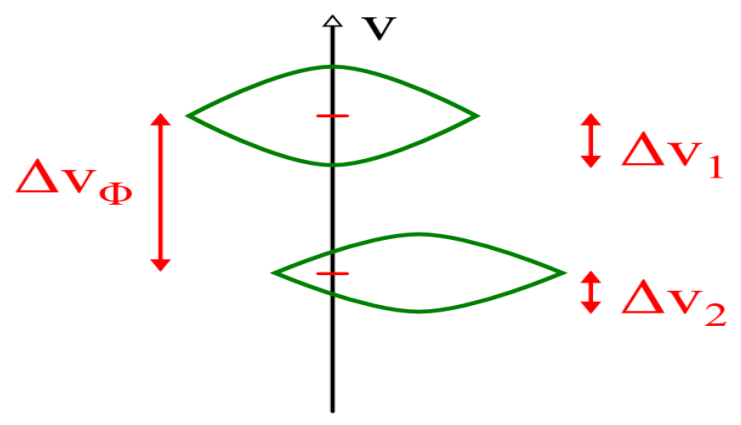

FIG. 6: Snapshot of the trapping domains of two nearby waves. They have half-widths $\Delta v_{i}$ and a mismatch in velocity $\Delta v_{\Phi}$.

\section{Diffusion in a given spectrum of waves}

Quasilinear theory aims at describing the self-consistent evolution of waves and particles. One of its final coupled equations is a diffusion equation with a diffusion coefficient computed through perturbation theory. Since during saturation particle dynamics is chaotic in the beam velocity domain, one may wonder about the validity of such a formula, even if the wave spectrum is prescribed. When investigating this issue, some surprises were on the way!

First, a single physical realization of the wave field acts on the particle velocity distribution to make it non gaussian, which rules out diffusion: "chaotic" does not mean "stochastic". One needs an ensemble of "enough" independent realizations to make it gaussian [10]. A simple way to do this is for waves to have mutually independent random phases.

The motion of a particle in a discrete set of waves with random phases involves several times. First, a discretization time $\tau_{\text {discr }}=(k \Delta v)^{-1}$ where $k$ is the typical wavenumber and $\Delta v$ is the typical mismatch of nearby phase velocities. Second, the already defined spreading time $\tau_{\text {spread }}$. Third, the autocorrelation time $\tau_{\text {ac }}=(k \Delta u)^{-1}$ where $\Delta u$ is the full range of phase velocities. In the weak warm beam case, $\Delta u$ is the width of the beam distribution function and $\tau_{\text {ac }}$ is the smallest of all three times ${ }^{40}$. The Chirikov overlap parameter [31] between two nearby waves is $s=\left(\Delta v_{1}+\Delta v_{2}\right) / \Delta v_{\Phi}$ where $\Delta v_{\Phi}$ is the mismatch of their phase velocities, and $\Delta v_{i}$ 's is the width in velocity of the trapping domain of wave $i$ which scales like the square root of the wave potential ${ }^{41}$ (see figure 6).

Whatever be the overlap of nearby resonances, perturbation theory is correct over a time $\tau_{\text {spread }}$. Therefore, initially particle dynamics looks diffusive and the diffusion coefficient takes on the quasilinear value. A small value of Chirikov overlap parameter $s$ is equivalent to $\tau_{\text {discr }} \ll \tau_{\text {spread }}$. Then at $t \simeq \tau_{\text {discr }}$ the particles feel they are in a quasiperiodic force field and the spreading of their velocities saturates. If the wave potential is periodic both in time and space, this saturation is due to the presence of KAM tori. In any case, till $\tau_{\text {discr }}$ particles feel the force field as a white noise and experience a stochastic diffusion. Figure $7(\mathrm{a})$ displays a cartoon of the variance $\left\langle\Delta v^{2}\right\rangle$ of the velocities of particles all released with the same initial velocity in a prescribed spectrum of Langmuir waves. The stochastic diffusion corresponds to the green segment on the left, and the saturation to the red segment on the right. As might be expected, chaos does not enter this picture.

When chaos becomes dominant, i.e. when $\tau_{\text {discr }} \gg \tau_{\text {spread }}$, numerical calculations revealed [30] that after a time $\tau_{s} \sim \tau_{\text {spread }},\left\langle\Delta v^{2}(t)\right\rangle$ grows with a slope in between the quasilinear one and 2.3 times this value ${ }^{42}$ (in the range

40 In the opposite limit when $\tau_{\text {spread }} / \tau_{\text {ac }}$ is small, the time evolution of the waves is slow with respect to the trapping motion in the instantaneous wave potential. Then chaotic dynamics may be described in an adiabatic way with the picture of a slowly pulsating separatrix [46, 47] (see also section 5.5 of [48] and 14.5.2 of [54]). In this limit, for the case of the motion in two waves, the resonance overlap defined hereafter is large.

41 This criterion is a very useful rule of thumb which works, also experimentally [38], provided the two trapping domains are not too dissimilar. In particular, $\Delta v_{1} / \Delta v_{2}$ should not be too far from 1 . Otherwise, one of the waves is a small perturbation for the other one, and the threshold of large scale chaos is a lot larger than 1 (see [52, 56] for more information). A more accurate way to understand the transition to large scale chaos is provided by a renormalization transformation [52, 56] (see also section 5.4 of [48] and 14.5.4 of [54]). However Chirikov criterion can also be used to check whether high dimensional dynamics is chaotic enough. More specifically parameter $s$ may be used as an observable whose Gibbsian estimate tells Gibbsian calculus makes sense when it is larger than 1 [62].

42 The necessity to go beyond $\tau_{\text {spread }}$ to see the chaotic diffusion is a caveat for the numerical measurement of a chaotic diffusion coefficient. 

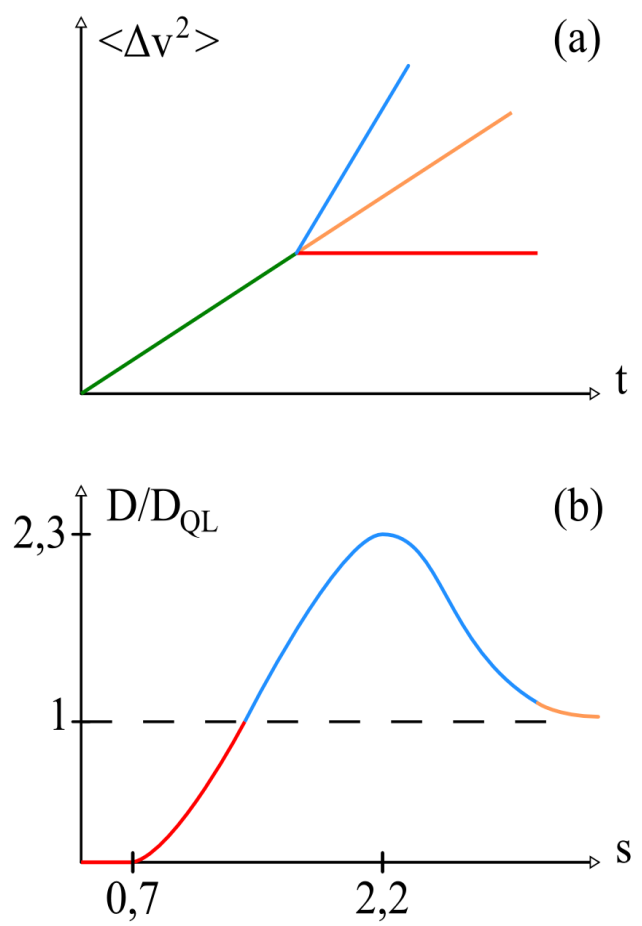

FIG. 7: Regimes of diffusion. (a) $\left\langle\Delta v^{2}\right\rangle$ vs. time; initial quasilinear regime: green line; asymptotic saturation: red line; superquasilinear regime: blue line; time-asymptotic quasilinear regime: brown line. (b) $D / D_{\mathrm{QL}}$ vs. $s$; same color code as in (a), except for the red growing segment that corresponds to the weakly chaotic regime.

bounded by the brown and blue curves in figure $7(\mathrm{a}))$.

Figure 7(b) summarizes in a sketchy way the various regimes as to the value of the diffusion coefficient $D$ measured

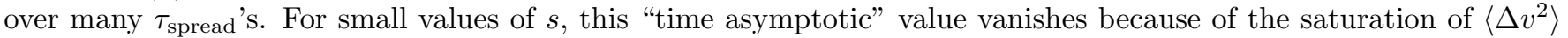
after $\tau_{\text {discr }}$. This corresponds to the horizontal red segment. When $s$ grows above the chaotic threshold, $D$ takes on positive values, but first below the quasilinear one (red growing curve in figure 7(b)). For intermediate values of $s, D$ takes on superquasilinear values (blue curve in figure $7(\mathrm{~b})$ ). For large values of $s, D$ takes on the quasilinear value ${ }^{43}$ (brown curve in figure $7(\mathrm{~b})$ ).

This can be understood by considering the dynamics of a particle in a prescribed spectrum of waves defined by Hamiltonian

$$
H(p, q, t)=\frac{p^{2}}{2}+A \sum_{m=\mu}^{M} \cos \left(m q-t+\varphi_{m}\right),
$$

where the $\varphi_{m}$ 's are random variables, and $M \gg \mu \gg 1$. Let the particle have an initial velocity $p_{0}$ in between $1 / m_{0}$ and $1 /\left(m_{0}+1\right)$, with $M \gg m_{0} \gg \mu$. We evaluate $\Delta p(t)=p(t)-p_{0}$ by integrating formally the equation of motion for $p$. For $t$ small enough, the dependence of $\Delta q=q(t)-p_{0} t-q_{0}$ on any two phases with all other phases fixed is

This minimum time comes from the locality in velocity of wave-particle interaction [10, 48]. Indeed it can be shown that at a given moment the waves making particle dynamics chaotic have a phase velocity within $\Delta v \sim 1 /\left(k \tau_{\text {spread }}\right)$ from the particle velocity. Those out of this range act perturbatively. If waves have random phases, after visiting several "resonance boxes" of width $\Delta v$, a particle feels as having been acted upon by a series of independent chaotic dynamics, which triggers a diffusive behavior. This decorrelation makes it possible to numerically measure the diffusion coefficient by following the dynamics either of a single particle for a series of random outcomes of the wave phases, or of many particles for a single typical outcome of the phases. By extension this enables to reconcile the uniqueness of each realization of an $N$-body system with models invoking a probabilistic average over independent realizations.

43 If the waves have random amplitudes $A_{m}$ and phases $\varphi_{m}$ such that $A_{m} \exp \left(\mathrm{i} \varphi_{m}\right)$ is a gaussian variable, then the superquasilinear bump does not exist, and $D / D_{\mathrm{QL}} \leq 1$ for all values of $s$, but still goes to 1 when $s$ becomes large [42]. 
weak. Then $\langle\Delta p(t)\rangle=0$. We write $\left\langle\Delta p^{2}(t)\right\rangle=\Delta_{0}+\Delta_{+}+\Delta_{-}$, with

$$
\Delta_{j}=-\epsilon_{j} A^{2} \int_{0}^{t} \int_{0}^{t} \sum_{m_{1}=\mu}^{M} \sum_{m_{2}=\mu}^{M} \frac{m_{1} m_{2}}{2}\left\langle\cos \left[\Phi_{m_{1}}\left(t_{1}\right)+\epsilon_{j} \Phi_{m_{2}}\left(t_{2}\right)\right]\right\rangle \mathrm{d} t_{1} \mathrm{~d} t_{2}
$$

where

$$
\Phi_{m}(t)=m \Delta q(t)+\Omega_{m} t+m q_{0}+\varphi_{m}
$$

where $\Omega_{m}=m p_{0}-1$, with $\epsilon_{ \pm}= \pm 1$ and $\epsilon_{0}=-1$, and under condition $m_{1} \neq m_{2}$ for $j=-$, and condition $m_{1}=m_{2}$ for $j=0$. Let $t_{-}=t_{1}-t_{2}$ and $t_{+}=\left(t_{1}+t_{2}\right) / 2$.

For $t_{-} \ll \tau_{\text {spread }},\left\langle\exp \left[\mathrm{i} k_{m}\left(\Delta q\left(t_{+}+t_{-} / 2\right)-\Delta q\left(t_{+}-t_{-} / 2\right)\right)\right]\right\rangle$ may be considered equal to 1 . Therefore the support in $t_{-}$of the integrand in $\Delta_{0}$ is on the order of $\tau_{\text {ac }}$. Since $\tau_{\mathrm{ac}} \ll \tau_{\text {spread }}$, the integration domain in $t_{-}$may be restricted to $\left|t_{-}\right| \leq \nu \tau_{\text {ac }}$ where $\nu$ is a few units. In the limit where $\nu \tau_{\text {ac }} \ll t \ll \tau_{\text {discr }}$, we obtain

$$
\begin{aligned}
\Delta_{0} & \simeq \sum_{m=\mu}^{M} \int_{0}^{t} 2 D_{\mathrm{QL}}\left(p_{0}\right) \pi^{-1} \int_{0}^{\nu \tau_{\mathrm{ac}}}\left\langle\cos \left[\Omega_{m} t_{-}\right]\right\rangle p_{0} \mathrm{~d} t_{-} \mathrm{d} t_{+} \\
& =2 D_{\mathrm{QL}}\left(p_{0}\right) \sum_{m=\mu}^{M}\left(\pi \Omega_{m}\right)^{-1}\left\langle\sin \left[\Omega_{m} \nu \tau_{\mathrm{ac}}\right]\right\rangle p_{0} t=2 D_{\mathrm{QL}}\left(p_{0}\right) t
\end{aligned}
$$

where the discrete sum over $m$ was approximated by an integral, and where $D_{\mathrm{QL}}\left(p_{0}\right)=\pi\left(A m_{0}\right)^{2} / p_{0}$ is the quasilinear diffusion coefficient. $\Delta_{ \pm}$can be neglected since we assumed $\Delta q$ to depend weakly on two phases with all other phases fixed. For small times, a particle feels a stochastic forcing due to many waves. Therefore its position has a weak dependence over any two random phases, which justifies the quasilinear estimate. If $s \gg 1$, it can be shown ([58], section 6.8.2 of [15], and Appendix 2) that the position of a particle has a weak dependence over any two random phases over a time on the order of $\tau_{\mathrm{QL}}=\tau_{\text {spread }} \ln (s)$. Therefore, if $s \gg 1$, the quasilinear estimate holds over a time $\tau_{\mathrm{QL}} \gg \tau_{\text {spread. }}$. Using this property, the estimate can be shown to be correct for all times ([58] and Appendix 2 ). This derivation of the quasilinear estimate in the $s \rightarrow \infty$ limit is not yet rigorous. However by using probabilistic techniques, a rigorous proof can be obtained for the dynamics of particles in a set of waves with the same wavenumber and integer frequencies, if their electric field is gaussian [49], or just if their phases have enough randomness [43].

We have just shown that in the resonance overlap regime $D / D_{\mathrm{QL}}$ may cover a large range of values [30, 48]. In particular $D \simeq D_{\mathrm{QL}}$ is obtained for random phases of the waves and strong resonance overlap [30, 43, 48, 49]. The plateau regime corresponds to $\gamma_{\mathrm{L}}=0$ and therefore to $\gamma_{\mathrm{L}} \tau_{\text {spread }}=0$. Since $D / D_{\mathrm{QL}}$ may cover a large range of values in this regime, $\gamma_{\mathrm{L}} \tau_{\text {spread }} \ll 1$ does not imply per se any renormalization or non-renormalization of $D / D_{\mathrm{QL}}$, nor of $\gamma / \gamma_{\mathrm{L}}$ by wave-particle momentum conservation. This contradicts previous works using $\gamma_{\mathrm{L}} \tau_{\text {spread }} \ll 1$ to try and prove the validity of quasilinear theory [48, 58, 90,91] and the "turbulent trapping" Ansatz aiming at the contrary [89]. The value of $D / D_{\mathrm{QL}}$ in the plateau regime of the bump-on-tail instability depends on the kind of wave spectrum the beam-plasma system reaches during the saturation of the instability, and not only on condition $\gamma_{\mathrm{L}} \tau_{\text {spread }} \ll 1$, as assumed by these works.

\section{E. A crucial numerical simulation}

In order to find out the nature of the wave spectrum at saturation, numerical simulations were performed using a semi-Lagrangian code for the Vlasov-wave model [15]. This model is the mean-field limit of the granular dynamics defined by the self-consistent Hamiltonian: waves are still present as $M$ harmonic oscillators, but particles are described by a continuous distribution function.

The simulations were benchmarked in various ways. In particular, they recovered that the wave spectrum is almost frozen when the plateau is formed. They were repeated for a large number of random realizations of the initial wave phases for a fixed initial spectrum of amplitudes. As shown by previous simulations, the final wave spectrum was found to be quite jaggy, and not smooth as that predicted by QL theory. For each of the realizations, one computed the spreading of the velocities of test particles when acted upon by the final set of waves. The first four even moments of this spreading were compared with those of the solution to the quasilinear Fokker-Planck equation for velocity diffusion, using the velocity-dependent diffusion constant $D_{Q L}$ computed with the final wave spectrum. The agreement was found to be excellent: the plateau verified the predictions of QL theory. However, as found in previous numerical simulations and experimentally, mode-mode coupling was found to be very strong during the saturation. 
At this point, the validity of QL predictions while QL assumptions are wrong sounded still like a mystery. However, the simulations brought an unexpected clue to elucidate it: the variation of the phase of a given wave with time was found to be almost non fluctuating with the random realizations of the initial wave phases [16]. Therefore the simulations showed that the randomness of the final wave phases was a mere consequence of that of initial phases. As a result, the self-consistent dynamics was shown to display an important ingredient for the validity of a quasilinear diffusion coefficient for the dynamics in a prescribed spectrum.

\section{F. New analytical calculations}

The just mentioned almost non fluctuating variation of the phase suggested to revisit the past analytical calculations of the wave phase and amplitude average evolutions. As mentioned at the end of section III A, they were performed by averaging over the initial particle positions. The new numerical result suggested to perform instead an average over the initial wave phases, which is compatible with a non uniform particle density. Furthermore, the previous calculations used a perturbative approach which made sense in the linear regime, but which might be unjustified for the chaotic regime of the instability. This was an incentive to use the Picard iteration technique which is the central tool to prove the existence and uniqueness of solutions to differential equations in the so-called Picard's existence theorem, Picard-Lindelöf theorem, or Cauchy-Lipschitz theorem ${ }^{44}$. The iteration turns out to be analytically tractable three times when starting from the ballistic solution. It can be shown analytically that the third order Picard iterate is able to describe the separation between trapped and passing orbits of a nonlinear pendulum. Furthermore numerical calculations [44] indicate that for the chaotic motion of particles in a prescribed set of waves such an iterated solution is already fairly good over the $\tau_{\text {spread }}$ time-scale which is crucial for chaos to build up. However the accuracy of the third order Picard iterate needs further assessment.

This work in progress already brings the following results. First, the modification of the average wave frequency due to the coupling with particles is exactly the principal part correction to the wave frequency provided by the Vlasovian calculation of the dispersion relation of Langmuir waves or by the equivalent calculations with the self-consistent Hamiltonian [48]. However, the latter calculations deal with a spatially uniform distribution of particles, while the present one holds whatever the spatial inhomogeneity of the distribution of tail particles, but requires an average over the phases of the Langmuir waves. Second, an estimate of phase fluctuations shows they scale like $\eta^{1 / 2}$, which makes them negligible, as shown by simulations. Therefore, if initial phases are random, they stay random for all times: there is no need for the traditional random phase approximation.

Third, assuming the wave spectrum of any realization to be smooth when averaged over a width in phase velocity on the order of $\left(k \tau_{\text {spread }}\right)^{-1}$, where $k$ is a typical wavenumber, the evolution of a wave amplitude $A_{j}$ is given by

$$
\frac{\mathrm{d}\left\langle\left|A_{j}\right|^{2}\right\rangle}{\mathrm{d} t}=2 \gamma_{j \mathrm{~L}}\left\langle\left|A_{j}\right|^{2}\right\rangle+S_{\mathrm{spont} j}+S_{\text {inhom } j},
$$

where $\gamma_{\mathrm{L}}$ is the Landau growth rate defined together with equation $(4), S_{\text {spont }} \sim \bar{f}\left(v_{\text {phase }}\right) / N$, where $\bar{f}$ now is the space averaged coarse-grained velocity distribution function of the tail particles, $v_{\text {phase }}$ is the phase velocity of the wave, and

$$
S_{\text {inhom } j}=\frac{N^{2} \varepsilon_{j}^{4}}{k_{j}^{2}} \int_{0}^{t} \mathrm{~d} t^{\prime} \iint \mathrm{d} p \mathrm{~d} p^{\prime} \mathrm{e}^{\mathrm{i}\left[\Omega_{j}\left(p^{\prime}\right) t^{\prime}-\Omega_{j}(p) t\right]}\left\langle\tilde{f}\left(-k_{j}, p^{\prime}, t^{\prime}\right) \tilde{f}\left(k_{j}, p, t\right)\right\rangle+\text { c.c. },
$$

where $\tilde{f}$ is the Fourier transform of the coarse-grained velocity distribution function, $\Omega_{j}(p)=k_{j} p-\omega_{j 0}$, and $t$ is on the order of $\tau_{\text {spread. }}$ Equation (9) displays successively the contribution to the wave amplitude evolution of Landau growth or damping, of spontaneous emission, and of the emission of spatial inhomogeneities (turbulent eddies). Because of the $1 / N$ factor, spontaneous emission vanishes when $N \rightarrow \infty$, since plasma graininess becomes negligible. To the contrary, the contribution of inhomogeneities to wave emission does not vanish in this limit. Due to turbulent trapping, a gradient in the velocity distribution yields localized spatial gradients a quarter of trapping time later, but this exchange of slopes in space and velocity occurs in a fluctuating way. If $f(x, p)$ does not depend on $x$, $S_{\text {inhom }}$ vanishes. This occurs in particular when the plateau forms at the end of the weak beam-plasma instability in the limit $N \rightarrow \infty$ [15]. If such an instability starts from a position-independent velocity distribution function, the $\tilde{f}$ 's are

44 This iteration technique is very convenient to alleviate the algebra of many perturbation calculations. This is the case for the perturbation calculation of the dynamics defined by Hamiltonian (5) in the wave amplitude $A$. In particular for a single wave, which provides equation 2 . 
only due to turbulent eddies. Then the size of $S_{\text {inhomj }}$ can be bounded by a quantity vanishing in the limit where the number of waves is large, i.e. for a continuous wave spectrum. Therefore, if these calculations make sense, in this limit the quasilinear equations might correctly describe the average behavior of the instability, even though a given realization be very far away from the average behavior.

\section{CONCLUSION}

The main messages of this chapter are summarized in the abstract and in Appendix 1, and are not recalled here. This short conclusive section is rather devoted to global remarks and prospects.

The part of this chapter devoted to wave-particle interaction in plasmas shows the description of collisionless plasmas as finite dimensional Hamiltonian systems is relevant, simple and transparent for linear aspects, powerful even for nonlinear and chaotic ones. It shows the irreversible evolution of a macroscopic system can be described by classical mechanics. Therefore an old dream comes true, but is yet to be made rigorous. As a result, the finite dimensional approach opens new avenues for the description of plasmas. However the mean-field description (Vlasov equation) stays a powerful tool, in particular for linear calculations, for exhibiting the metastable BGK modes, and for numerical simulations.

The description of complexity of plasmas is an intricate issue, and even more so the future development of the corresponding methodology. Collective effects are important in plasma physics, but also for its development: it requires collective efforts of plasma physicists. Therefore this chapter is more a way to start a brainstorming in the plasma community than a list of ready-to-use recipes. It would be very useful for this community to pay attention to the essence of its physics and of its practice. To this end it should review, retrace, and revisit past published material, but also its past way of thinking, of interacting, and of meeting together. Such an attentive attitude is reminiscent of Cicero's quite philosophical proposal of the right way for the Roman citizens to be religious, linking it to elegance, diligence, and intelligence ${ }^{45}$ : a nice inspiration for the work to come!

Acknowledgements: I am indebted to Y. Camenen, L. Couëdel, F. Doveil, and Y. Elskens, for a thorough reading of a first version of this paper and for providing me with an extensive feedback. My thanks also go to D. Bonfiglio, S. Cappello, and F. Sattin who did the same for a second version. Y. Elskens also helped me a lot in improving the English. F. Baldovin, M. Bécoulet, D. Bénisti, N. Bian, A. Boozer, P. Diamond, M.-C. Firpo, M. Henneaux, T. Mendonça, B. Momo, K. Razumova, S. Ruffo, M. Valisa, and F. Zolla are thanked for very useful comments and new references. I thank D. Guyomarc'h for drawing all the figures. My thanks go to M. Farge who pointed out to me reference [112]. The topic of my talk at Chaos, Complexity and Transport 2011 was about the description of self-consistent wave-particle interaction with a finite-dimensional Hamiltonian described in section 3 . However, two seminars I gave later on in the north and south campuses of Marseilles were the occasion to start developing the ideas of section 2, in kind of an echo to section III. I thank the organizers of the conference for allowing me to extend the topic of my chapter beyond the original contents of my talk, and to further develop my thoughts about plasma complexity and the way to tackle it.

\section{APPENDIX 1: EXTENDED SUMMARY}

The introduction recalls what are plasmas and provides a definition of complexity relevant to plasma physics. The chapter has two main parts. The first one is subjective and aims at favoring a brainstorming in the plasma community. It discusses the present theoretical description of plasmas, with a focus on hot weakly collisional plasmas. One of the purposes of this paper is to stop and to look backward to proceed better ahead. How do we work? How could our community improve its methodology? The first part of this chapter (section II) is made up of two sub-parts. The first one (section II A) deals with the present status of this description, while the second one (section II B) considers possible methodological improvements, some of them specific to plasma physics, but many may be of possible interest for other fields of science. The second part of this chapter (section III) is devoted to one instance where modern

\footnotetext{
45 "Qui autem omnia, quae ad cultum deorum pertinerent, diligenter retractarent et tamquam relegerent, sunt dicti religiosi ex relegendo, ut elegantes ex eligendo, ex diligendo diligentes, ex intellegendo intellegentes; his enim in verbis omnibus inest vis legendi eadem quae in religioso." Cicero, De Natura Deorum, 2, 28. English translation [33]: "Those on the other hand who carefully reviewed and so to speak retraced all the lore of ritual were called "religious" from relegere (to retrace or re-read), like "elegant" from eligere (to select), "diligent" from diligere (to care for), "intelligent" from "intellegere" (to understand) ; for all these words contain the same sense of "picking out" (legere) that is present in "religious"".
} 
nonlinear dynamics and chaos helped revisiting and unifying the overall presentation of a paradigm of wave-particle interaction in plasmas.

Section 2.1, devoted to the present status of the description of plasma complexity, first recalls the path used for training students to this complexity. Then it recalls that most models used in plasma physics, even the Vlasov equation, have feet of clay, since they cannot be derived in an axiomatic way from first principles with conditions of validity suited to their actual applications. Each plasma physicist is shown to elaborate his own global view about plasma physics from many models which do not have any strict hierarchy. A principle of simplicity (Occam's razor principle) dominates the modeling activity. The validation of assumptions turns out to be more difficult for a complex system than for a simple one, because of the lack of information about it. In agreement with Popper's paradigm at any moment the description of plasma complexity is provisional. It results from a collective and somewhat unconscious process. This makes changing views more difficult. Numerical simulations are discussed as a complex tool to face complexity. However the complexity they describe is still much smaller than in actual experiments, they often come without error bars on their predictions, the numerical coding of an analytical model often involves many uncontrolled approximations, and the role of intentionality is higher than in analytical calculations because of the choice of initial conditions and of parameters. Examples are provided at the various steps of this section.

Section 2.2, devoted to possible methodological improvements, motivates them by stating difficulties faced by plasma physicists, like information retrieval, the inflation of publications and the growing importance of oriented programs. Working on complex systems is a hard task, but the present trend of scientific practice makes it even harder. Therefore plasma physicists would gain very much in any improvement of this practice, and might be motivated into impelling a change. The proposals for improving the present situation go along the following lines: improving the ways papers are structured, improving the way scientific quality is assessed in the referral process, developing new data bases, stimulating the scientific discussion of published results, diversifying the way results are made available, assessing more quality than quantity, making available an incompressible time for creative thinking and non purpose-oriented research. Some possible improvements for teaching are also indicated.

The suggested improvement to the structure of papers is the following: each paper, even letters, would have a "claim section" being a kind of executive summary. It would summarize the main results and their most relevant connection to previous literature. It would provide a clear information about the importance, the originality, the actual scientific contribution of the paper, and about the "precedents, sources, and context of the reported work" as worded in the APS guidelines for professional conduct. Salient figures or formulas would be set there to support the claims. This procedure would improve the clarity of the papers by driving an author to state the essence of his results in a more accessible way, and without having to care about the literary constraints of a normal text.

With this tool, the referral process might be improved by requiring referees to check the claims of the claim section, and to motivate their possible disagreements with any of them. This procedure should make the referral process more scientifically rigorous, more ethical, and faster. Editors would benefit from a better refereeing process, which would avoid many authors' complaints, while accelerating the editorial process. Journals would benefit from the increased clarity of the contents of their published papers. The procedure might start with an experimental stage where the claim section would be optional for the authors, but not for the referees if the claim section is available.

The claim sections might be set by each scientific journals or publisher into a new dedicated data base accessible through Internet where cross-referenced papers would be hyperlinked. This would provide a new technique for data retrieval adapted to plasma complexity. It would ease the assessment of the state of the art of a given topic, with respect to what is available through present bibliographical databases.

One might consider broadening the way papers are commented in journals by adding a first friendlier step where a direct contact with the authors would lead to publishing a common short corrective communication, naturally linked to the original claim section of the original paper. A classical comment to the journal would be sent only if the authors could not agree about a common view.

As to possible improvements for teaching, student should be made conscious about the limited capabilities of models. One may avoid teaching many calculations to start with, but keep the physical ideas and the corresponding images. Nonlinear dynamics and chaos might provide a way to revisit and unify separated chapters, e.g. turbulent and "collisional transport", the calculation of magnetic field lines, or the introduction of fluid and of Vlasov equations.

The second part of this chapter (section III) is more specialized, and is a scientific presentation of a theoretical approach avoiding several shortcomings of the Vlasovian approach. It deals with Langmuir wave-electron interaction in one-dimensional plasmas. This topic is tackled by describing plasma dynamics with a finite dimensional Hamiltonian system coupling $N$ particles with $M$ waves, the self-consistent Hamiltonian. This enables recovering Vlasovian linear theory with a mechanical understanding. In particular, the reason why Landau damping cannot be an eigenmode is shown to be rooted deeply in Hamiltonian mechanics. This damping is recovered as an analogue of van Kampen phase-mixing effect. This phase-mixing in turn plays an essential role in the calculation of Landau instability. The self-consistent dynamics reveals that both Landau damping and instability result from the same synchronization mechanism of particles with waves. 
The quasilinear description of the weak warm beam, or bump-on-tail, instability is then recalled, together with the apparent paradox that its predictions look correct while its assumptions are proved to be wrong. A recent analytical result shows that self-consistency vanishes when the plateau forms in the tail distribution function: the wave spectrum is frozen. This leads to consider the dynamics of particles in a frozen spectrum of waves with random phases. It involves a fundamental time-scale, the spreading time $\tau_{\text {spread }}$ after which the positions of particles are spread by a typical wave-length of the waves with respect to their ballistic values. Till a time at most $\tau_{\text {spread }}$, particles feel the global force due to the waves as a stochastic force, and their velocities diffuse in a quasilinear way. If their dynamics is not chaotic, they eventually feel the quasi-periodic nature of the force, and diffusion stops. If their dynamics is chaotic, after a time $\tau_{\text {spread }}$, they experience a chaotic diffusion that may be superquasilinear by a factor 2.3 , but which becomes quasilinear in the limit of strong resonance overlap. The latter result is understood as a consequence of the weak dependence of the particle dynamics over any two phases over a time much larger than $\tau_{\text {spread }}$.

Then is recalled a recent numerical simulation of the bump-on-tail instability aiming at checking whether diffusion is quasilinear when the plateau sets in, and proving that it is indeed. It also brought the unexpected result that the variation of the phase of a given wave with time is almost not fluctuating for random realizations of the initial wave phases. This was an incentive to undertake new analytical calculations of the average behavior of the self-consistent dynamics when the initial wave phases are random. Using Picard iteration technique, they show that the modification of the average wave frequency due to the coupling with particles is exactly the principal part correction to the wave frequency provided by the Vlasovian calculation of the dispersion relation of Langmuir waves or by the equivalent calculations with the self-consistent Hamiltonian [48]. However the latter calculations deal with a spatially uniform distribution of particles, while the present one holds whatever the spatial inhomogeneity of the distribution of tail particles. An estimate of phase fluctuations shows they are negligible, confirming simulation results. The evolution of the wave amplitude involves the Landau effect and spontaneous emission, as already found for a spatially uniform distribution of particles, and a "spontaneous emission" of spatial inhomogeneities.

\section{APPENDIX 2: FIRST EXAMPLE OF A CLAIM SECTION}

Here is proposed a claim section for paper [58] quoted in section III D. The title of the paper is: "Proof of quasilinear equations in the chaotic regime of the weak warm beam instability", and its abstract is: "The diffusion coefficient is proved rigorously to take on the quasilinear value for the chaotic motion of an electron in a prescribed set of strongly overlapping Langmuir waves with random phases. Natural approximations show this result to extend to the self-consistent chaotic motion of many particles in a set of many Langmuir waves corresponding to the weak warm beam instability. The weak influence of any particle on any wave and vice-versa is an essential ingredient of the derivation. Wave-particle momentum conservation implies the Landau growth rate to be related to the quasilinear diffusion coefficient." A possible claim section follows.

One considers the one-dimensional chaotic motion of an electron in a prescribed set of $M \gg 1$ strongly overlapping Langmuir waves with random phases and a regular enough spectrum. Let $k$ be the typical wavenumber of a wave, $q_{0}$ and $p_{0}$ be the initial particle position and velocity, and $q(t)$ its position at time $t$. Let $\Delta q(t)=q(t)-q_{0}-p_{0} t$.

Claim 1: The variation of $k \Delta q(t)$ with any two phases stays small with respect to $2 \pi$ over a time on the order of $\tau_{\mathrm{QL}}=\tau_{\text {spread }} \ln (s)$, where $s \gg 1$ is the typical resonance overlap parameter of two nearby waves in the spectrum, and $\tau_{\text {spread }}=4\left(k^{2} D_{\mathrm{QL}}\right)^{-1 / 3}$, with $D_{\mathrm{QL}}$ the typical value of the quasilinear diffusion coefficient.

Claim 2: This implies the particle diffuses in a quasilinear way up to a time on the order of $\tau_{\mathrm{QL}}$.

Claim 3: The latter property implies the particle diffuses in the same way for larger times.

Claim 4: The same property holds for the self-consistent dynamics defined by Hamiltonian (1) provided the wave spectrum be regular enough too.

Most relevant connection to previous literature: [10, 30, 40, 89, 120].

\section{APPENDIX 3: SECOND EXAMPLE OF A CLAIM SECTION}

Here is proposed a claim section for paper [61] quoted in section II A 4. The title of the paper is: "Calculation of transport coefficient profiles in modulation experiments as an inverse problem", and its abstract is: "The calculation of transport profiles from experimental measurements belongs in the category of inverse problems which are known to come with issues of ill-conditioning or singularity. A reformulation of the calculation, the matricial approach, is proposed for periodically modulated experiments, within the context of the standard advection-diffusion model where these issues are related to the vanishing of the determinant of a $2 \times 2$ matrix. This sheds light on the accuracy of calculations with transport codes, and provides a path for a more precise assessment of the profiles and of the related uncertainty." A possible claim section follows. 
One applies the advection-diffusion model for the quantity $\zeta(r, t)$

$$
\begin{aligned}
& \partial_{t} \zeta=-\nabla \cdot \Gamma(\zeta)+S \\
& \Gamma=-\chi \nabla \zeta+V \zeta
\end{aligned}
$$

to modulation experiments. One considers cylindrical symmetry and a purely sinusoidal forcing term $S$ with pulsation $\omega$.

Claim 1: Decomposing the signal $\zeta$ into a real amplitude and phase, $\zeta=A e^{i \phi}$, and $S$ as $S=S_{r}+i S_{i}$, yields

$$
\begin{aligned}
& \mathbf{M} \cdot \mathbf{Y}=\mathbf{G} \\
& \mathbf{Y}=\left(\begin{array}{c}
\chi \\
V
\end{array}\right), \quad \mathbf{M}=\left[\begin{array}{cc}
-A^{\prime} \cos \phi+A \phi^{\prime} \sin \phi & A \cos \phi \\
-A^{\prime} \sin \phi-A \phi^{\prime} \cos \phi & A \sin \phi
\end{array}\right], \\
& \mathbf{G}=\left[\begin{array}{c}
\frac{1}{r} \int_{0}^{r} d z z\left(S_{r}(z)-\omega A(z) \sin \phi(z)\right) \\
\frac{1}{r} \int_{0}^{r} d z z\left(S_{i}(z)+\omega A(z) \cos \phi(z)\right)
\end{array}\right]
\end{aligned}
$$

where the primes stand for differentiation with respect to $r$, and where all quantities in the 1 . h. s. of the first equation are computed at radius $r$.

Claim 2: On this basis, and with a controllable smoothing of the experimental data, the profile of transport coefficients is computed by inverting matrix $\mathbf{M}(r)$ at each measurement point.

Claim 3: This method enables a precise estimate of the uncertainty on the transport coefficients from that on the measurements at each measurement point.

Claim 4: The smaller the uncertainty on the estimate of the derivatives of $A$ and $\phi$, the larger the precision in the reconstruction of transport profiles.

Claim 5: At a given $r$, the smaller the absolute value of an eigenvalue, the larger the uncertainty of $\mathbf{Y}(r)$ along the corresponding eigenvector of matrix $\mathbf{M}(r)$ for a given uncertainty on measured data for all radii.

Claim 6: This method is lighter computationally than classical transport codes.

Claim 7: The reconstruction radius-by-radius enables to see how different the uncertainties are over $\mathbf{Y}(r)$ as a function of $r$.

Claim 8: This uncertainty is larger in the regions where sources or sinks are present.

Claim 9: In contrast with transport codes, this method requires a single boundary condition only.

Most relevant connection to previous literature: [113]

[1] M. Antoni, Y. Elskens, D.F. Escande, Phys. Plasmas 5, 841 (1998)

[2] N. Arcis, D.F. Escande, M. Ottaviani, Phys. Lett. A347, 241 (2005)

[3] N. Arcis, D.F. Escande, M. Ottaviani, Phys. Plasmas 13, 052305 (2006)

[4] S.D. Baalrud, J.D. Callen, C.C. Hegna, Phys. Plasmas 15, 092111 (2008)

[5] S.D. Baalrud, J.D. Callen, C.C. Hegna, Phys. Plasmas 17, 055704 (2010)

[6] D.R. Baker, N.R. Ahern, A.Y. Wong, Phys. Rev. Lett., 20, 318 (1968)

[7] R. Balecu, Statistical dynamics Matter out of equilibrium, (World scientific, Singapore, 1993)

[8] J. Barré, et al., Phys. Rev. E69, 045501 (2004); J. Stat. Phys. 119, 677 (2005)

[9] J. Barré, et al., J. Stat. Phys. 119, 677 (2005)

[10] D. Bénisti, D.F. Escande, Phys. Plasmas 4, 1576 (1997)

[11] D. Bénisti, L. Gremillet, Phys. Plasmas 14, 042304 (2007)

[12] D. Bénisti, D.J. Strozzi , L. Gremillet, Phys. Plasmas 15, 030701 (2008)

[13] D. Bénisti, O. Morice, L. Gremillet, D.J. Strozzi , Transport Theory and Statistical Physics 40, 185 (2011)

[14] I.B Bernstein, J.M. Greene, M.D. Kruskal, Phys. Rev. 108, 546 (1957)

[15] N. Besse, Y. Elskens, D.F. Escande, P. Bertrand, Plasma Phys. Control. Fusion 53, 025012 (2011)

[16] N. Besse, Y. Elskens, D.F. Escande, P. Bertrand, Proc. 38th EPS Conference on Controlled Fusion and Plasma Physics, Strasbourg, 2011, P2.009

[17] D.D. Biskamp, Nonlinear Magnetohydrodynamics, (Cambridge University Press, Cambridge, 1993)

[18] D. Bonfiglio, D. F. Escande, P. Zanca, S. Cappello, Nucl. Fusion 51, 063016 (2011)

[19] D. Bonfiglio, S. Cappello, D. F. Escande, Phys. Rev. Lett. 94, 145001 (2005).

[20] W.J. Bos, R. Rubinstein, L. Fang, http://hal.archives-ouvertes.fr/hal-00605446/en/

[21] A. Campa, T. Dauxois, S. Ruffo, Phys. Reports 480, 57 (2009)

[22] S. Cappello, R. Paccagnella, in Proc. Workshop on Theory of Fusion Plasmas, ed. by E. Sindoni, (Compositori,Bologna, 1990), p. 595 
[23] S. Cappello, R. Paccagnella, Phys. Fluids B4, 611 (1992)

[24] S. Cappello, D. Biskamp, Proc. International Conf. on Plasma Phys. Nagoya, 1, 854 (1996)

[25] S. Cappello et al., Nucl. Fusion 51, 103012 (2011)

[26] S. Cappello, D. F. Escande, Phys. Rev. Lett. 85, 3838 (2000)

[27] S. Cappello et al., Theory of Fusion Plasmas, AIP Conference Proceedings 1069, 27 (2008)

[28] D. del-Castillo-Negrete, Proceedings of the First ITER Summer School. Turbulent Transport in Fusion Plasmas, ed. by S. Benkadda, AIP Conference Proceedings 1013, 207 (2008)

[29] J.R. Cary, R. Littlejohn, Annals of Physics 151, 1 (1983)

[30] J.R. Cary, D.F. Escande, A.D. Verga, Phys. Rev. Lett. 65, 3132 (1990)

[31] B.V. Chirikov, Phys. Rep. 52, 263 (1979)

[32] Long-range interacting systems, ed. by T. Dauxois, S. Ruffo, L.F. Cugliandolo, (Oxford University Press, Oxford, 2010)

[33] M. T. Cicero, De Natura Deorum, in De Natura Deorum Academica with an English Translation by H. Rackham, (Harvard University press, Cambridge , MA, 1967) p. 193, http://ia600302.us.archive.org/27/items/denaturadeorumac00ciceuoft/denaturadeorumac00ciceuoft.pdf

[34] L. de Broglie, Revolution in Physics, (Routledge and Kegan Paul, London, 1954).

[35] D. del-Castillo-Negrete, Proceedings of the First ITER Summer School. Turbulent Transport in Fusion Plasmas, ed. by S. Benkadda, AIP Conference Proceedings 1013, 207 (2008)

[36] D. del-Castillo-Negrete, Nonlinear processes in Geophysics 17, 795 (2010)

[37] F. Doveil, Y. Vosluisant, S.I. Tsunoda, Phys. Rev. Lett. 69, 2074 (1992)

[38] F. Doveil et al., Phys. Plasmas 12, 010702 (2005)

[39] F. Doveil, D.F. Escande, A. Macor, Phys. Rev. Lett., 94, 085003 (2005)

[40] W.E. Drummond, D. Pines, Nucl. Fusion Suppl. 3, 1049 (1962)

[41] K. Elsässer, Plasma Phys. Control. Fusion 28, 1743 (1986)

[42] Y. Elskens, Commun. Nonlinear Sci. Numer. Simul. 15, 10 (2010)

[43] Y. Elskens, submitted (2011)

[44] Y. Elskens, private communication (2011)

[45] Y. Elskens, ESAIM Proc. 10, 211 (2001), ed. by F. Coquel, S. Cordier, http://www.emath.fr/Maths/Proc

[46] Y. Elskens, D.F. Escande, Nonlinearity 4, 615 (1991)

[47] Y. Elskens, D.F. Escande, Physica D 62, 66 (1993)

[48] Y. Elskens, D.F. Escande, Microscopic dynamics of plasmas and chaos, (IoP, Bristol, 2003)

[49] Y. Elskens, E. Pardoux, Ann. Appl. Prob. 20, 2022 (2010)

[50] D.F. Escande, Phys. Rev. Lett. 35, 995 (1975)

[51] D.F. Escande, J.P.M. Schmitt, Phys. of Fluids 19, 1757 (1976).

[52] D.F. Escande, Phys. Rep. 121, 165 (1985)

[53] D.F. Escande, in Large scale structures in nonlinear physics, ed. by J.D. Fournier P.L. Sulem, Lect. Notes Phys. 392,73 (1991)

[54] D.F. Escande, in Long-range interacting systems, ed. by T. Dauxois, S. Ruffo, L.F. Cugliandolo, (Oxford University Press, Oxford, 2010), p. 469

[55] D.F. Escande, Proc. International Symposium on Waves, Coherent, Structures and Turbulence in Plasmas, AIP Conference Proceedings 1308, 85 (2010)

[56] D.F. Escande, F. Doveil, J. Stat. Phys. 26, 257 (1981)

[57] D.F. Escande, B. Souillard, Phys. Rev. Lett. 52, 1296 (1984)

[58] D.F. Escande, Y. Elskens, Phys. Lett. A302, 110 (2002)

[59] D.F. Escande, M. Ottaviani, Phys. Lett. A323, 278 (2004)

[60] D.F. Escande, F. Sattin, Phys. Rev. Lett. 99, 185005 (2007)

[61] D.F. Escande F. Sattin, Phys. Rev. Lett. 108, 125007 (2012)

[62] D.F. Escande, H. Kantz, R. Livi, S. Ruffo, J. Stat. Phys. 76, 605 (1994)

[63] D.F. Escande, S. Zekri, Y. Elskens, Phys. Plasmas 3, 3534 (1996)

[64] D. F. Escande, P. Martin, S. Ortolani et al., Phys. Rev. Lett. 85, 1662 (2000)

[65] D. F. Escande, R. Paccagnella et al., Phys. Rev. Lett. 85, 3169 (2000)

[66] W. Ettoumi and M.-C. Firpo, Phys. Rev. E84, 030103 (2011)

[67] J.M. Finn, R.A. Nebel, C.C. Bathke, Phys. Fluids B4, 1262 (1992)

[68] M.-C. Firpo, Y. Elskens, Phys. Rev. Lett. 84, 3318 (2000)

[69] M.-C. Firpo et al., Phys. Rev. E64, 026407 (2001)

[70] M.-C. Firpo, F. Leyvraz, G. Attuel, Phys. Plasmas 13, 122302 (2006)

[71] J.P. Freidberg, Ideal Magneto-Hydro-Dynamics, (Plenum Press, New York, 1987)

[72] B. Gallet et al., Phys. Rev. Lett. 108, 144501 (2012)

[73] S. Gasiorowicz, M. Neuman, R.J. Riddell, Phys. Rev. 101, 922 (1956)

[74] M. Gobbin et al., Phys. Rev. Lett. 106, 025001 (2011)

[75] M. Greenwald, Plasma Phys. Contr. Fus. 44, R27 (2002)

[76] R.J. Hastie, F. Militello, F. Porcelli, Phys. Rev. Lett. 95, 065001 (2005)

[77] R.D. Hazeltine, J.D. Meiss, Plasma Confinement, (Dover Press, Mineola, 2003)

[78] R.D. Hazeltine, F.L. Waelbroeck, The Framework of Plasma Physics, (Westview Press, Boulder, 2004)

[79] T.C. Hender et al., Nucl. Fusion 47, S128 (2007) 
[80] W. Horton, Rev. Mod. Phys., 71, 735 (1999)

[81] K. Ikeda et al., Nuclear Fusion 47, S1 (2007)

[82] B.B. Kadomtsev, Tokamak plasma: a complex physical system, (IOP, Bristol, 1992), p. 3

[83] N.G. van Kampen, Physica 21, 949 (1955)

[84] M.K.H. Kiessling, T. Neukirch, Proc. Natl. Acad. Sci. 100, 1510 (2003)

[85] C. Krafft, A. Volokitin, A. Zaslavsky, Phys. Rev. E82, 066402 (2010)

[86] R. Kraichnan, R. Panda, Phys. Fluids 31, 2395 (1988)

[87] T.S. Kuhn, The Structure of Scientific Revolutions, (University of Chicago Press, Chicago, 1996, first edition 1962)

[88] L.D. Landau, Zh. Eksp. Teor. Fiz. 16574 (1946); transl. J. Phys. USSR 10, 25 (1946); reprinted in Collected papers of Landau, ed. by D. ter Haar, (Pergamon, Oxford, 1965)

[89] G. Laval, D. Pesme, Phys. Rev. Lett. 53, 270 (1984)

[90] Y.-M.Liang, P.H. Diamond, Comments Plasma Phys. Controlled Fusion 15, 139 (1993)

[91] Y.-M.Liang, P.H. Diamond, Phys. Fluids B5, 4333 (1993)

[92] R. Lorenzini et al., Nature Phys. 5, 570 (2009)

[93] J.H. Malmberg, C.B. Wharton, Phys. Rev. Lett. 13184 (1964)

[94] F. Militello, F. Porcelli, Phys. Plasmas 11, L13 (2004)

[95] F. Militello, R.J. Hastie, F. Porcelli, Phys. Plasmas 13, 112512 (2006)

[96] R. Monchaux et al., Phys. Fluids 21, 035108 (2009)

[97] C. Mouhot, C. Villani, J. Math. Phys. 51, 015204 (2010)

[98] I.N. Onishchenko et al., ZhETF Pis. Red. 12, 407 (1970); transl. JETP Lett. 12, 281 (1970)

[99] T.M. O’Neil, Phys. Fluids 8, 2255 (1965)

[100] T.M. O’Neil, J.H. Winfrey, J.H. Malmberg, Phys. Fluids 4, 1204 (1971)

[101] S. Ortolani, D.D. Schnack, Magnetohydrodynamics of Plasma Relaxation, (World Scientific, Singapore, 1993)

[102] A.G. Peeters et al, Nucl. Fusion 51, 094027 (2011)

[103] F.W. Perkins et al., Nuclear Fusion 39, 2137 (1999)

[104] E. Piña, T. Ortiz, J. Phys. A: Math. Gen. 21, 1293 (1988)

[105] K.R. Popper, The Logic of Scientific Discovery, (Routledge, London, 2002)

[106] M.E. Puiatti et al., Phys. Plasmas 16, 012505 (2009)

[107] M.E. Puiatti et al., Nucl. Fusion 49, 045012 (2009)

[108] C. Roberson K.W. Gentle, Phys. Fluids 14, 2462 (1971)

[109] Yu.A. Romanov, G.F. Filippov,F Zh. Eksp. Theor. Phys. 40, 123 (1961); transl. Soviet Phys. JETP 13, 87 (1961)

[110] M.N. Rosenbluth, W.M. MacDonald, D.L. Judd, Phys. Rev. 107, 1 (1957)

[111] M.G. Rusbridge, Plasma Phys. Contr. Fus. 33, 1381 (1991)

[112] B. Russell, The scientific outlook, (George Allen and Unwin, London, 1931), part I, chapter II

[113] F. Ryter, R. Dux, P. Mantica, T. Tala, Plasma Phys. Contr. Fus. 52, 124043 (2010)

[114] P.B. Snyder et al., Nuclear Fusion 47, 961 (2007)

[115] H. Spohn, Large scale dynamics of interacting particles, (Springer, Berlin, 1991)

[116] J.B. Taylor, Phys. Rev. Lett. 33, 1139 (1974)

[117] J.L. Tennyson, J.D. Meiss, P.J. Morrison, Physica D 71, 1 (1994)

[118] P.W. Terry et al., Phys. Plasmas 15, 062503 (2008)

[119] S.I. Tsunoda, F. Doveil J.H. Malmberg, Phys. Fluids B3, 2747 (1991)

[120] A.A. Vedenov, E.P. Velikhov, R.Z. Sagdeev, Nucl. Fusion Suppl. 2, 465 (1962)

[121] J.A. Wesson, Tokamaks, (Oxford University press, Oxford, 2004)

[122] P.H. Yoon, T. Rhee, and C.-M. Ryu, Phys. Rev. Lett. 95, 215003 (2005)

[123] L.F. Ziebell, P.H. Yoon, J. Pavan, R. Gaelzer, Astrophys. J. 727, 16, (2011)

[124] http://slow-science.org/

[125] http://www.aps.org/policy/statements/02_2.cfm

[126] http://public.lanl.gov/alp/plasma/people/alfven.html

[127] http://www.iter.org/proj/iterhistory

[128] http://www.ias.edu/about/mission-and-history

[129] http://www.articleofthefuture.com/?utm_source=ESJ001\&utm_campaign=\&utm_content =\&utm_medium=email\&bid=UA81L4

[130] http://www.quantiki.org/wiki/Quantiki:About 